\title{
Classical limit of large $N$ gauge theories with conformal symmetry
}

\section{Seiji Terashima}

Yukawa Institute for Theoretical Physics, Kyoto University, Kitashirakawa Oiwakecho, Sakyo-ku, Kyoto 606-8502, Japan

E-mail: terasima@yukawa.kyoto-u.ac.jp

ABSTRACT: In this paper we study classical limit of conformal field theories realized by large $N$ gauge theories using the generalized coherent states. For generic large $N$ gauge theories with conformal symmetry, we show that the classical limit of them is described by the classical Einstein gravity. This may be regarded as a kind of derivation of the AdS/CFT correspondence.

Keywords: AdS-CFT Correspondence, Conformal Field Theory, 1/N Expansion, Field Theories in Higher Dimensions

ArXiv ePrint: 1907.05419 


\section{Contents}

1 Introduction and summary 1

2 Algebra for the energy momentum tensor $\quad 3$

2.1 Scalar case 3

2.2 OPE and the commutator algebra 5

$\begin{array}{lll}2.3 & \text { Algebra for the energy momentum tensor } & 9\end{array}$

3 Classical limit of the $C F T_{d} \quad 15$

4 Classical gravity on asymptotic $A d S_{d+1}$ and the $C F T_{d} \quad 18$

A Classical limit of $A d S / C F T$ and coherent state $\quad 20$

$\begin{array}{lll}\text { A.1 Coherent states for the linearized gravity } & 21\end{array}$

A.2 Coherent states for (3.2) 22

\section{Introduction and summary}

According to the AdS/CFT correspondence [1], a certain class of $d$-dimensional conformal field theories $\left(C F T_{d}\right)$ correspond to $d+1$-dimensional quantum gravity theory on an asymptotically $A d S_{d+1}$ spacetime. This conjecture has been investigated intensively and there are many evidences for this conjecture, although there is no proof. The explicit relation of the $A d S / C F T$ correspondence is the GKPW relation $[2,3]$ where a CFT partition function with the source terms is identified with the partition function of a quantum gravity on AdS with appropriate boundary conditions corresponding to the source terms. The extrapolation formula [4], which state that the boundary value of the bulk field is the CFT primary field, can be used as an explicit relation between the two theories. These are the "dictionaries" of the AdS/CFT correspondence and the basic assumptions of the most of the studies.

Alternatively, we can say that the $A d S / C F T$ correspondence is the equivalence of the two theories as a quantum theory. More explicitly, the correspondence means that the Hilbert spaces and the Hamiltonians of the two theories in the operator formalism are equivalent. ${ }^{1}$ In this formulation, a proof of the AdS/CFT correspondence means showing the spectrum of $C F T_{d}$ is equivalent to the spectrum of a quantum gravity on asymptotic $A d S_{d+1}$.

In [5], instead of assuming such dictionaries or existence of a bulk dual, we studied the (low energy) spectrum of generic large $N$ gauge theories with conformal symmetry, in the leading order in the large $N$ limit, and found that it is identical to the spectrum

\footnotetext{
${ }^{1}$ For this, we need to choose a time direction and the usual choice is the $C F T_{d}$ on $\mathbf{R} \times S^{d-1}$ where $\mathbf{R}$ represents the time. Note that in this formalism we can consider the sourceless case, which is simpler.
} 
of the Einstein gravity theory in global $A d S_{d+1}$ in the free theory limit, following some earlier works $[4,6,7]$ (see also [8]-[19]). Here, "generic" means that the theories satisfy the following two properties. The first one is that the low energy spectrum is determined only by the conserved symmetry currents whose conformal dimension is protected against the quantum corrections. ${ }^{2}$ In this paper, we further assume that the symmetry of the $C F T_{d}$ is the conformal symmetry only, for simplicity. The second one is that the states generated from the symmetry currents acting on the vacuum are completely independent except the relations imposed by the symmetry. ${ }^{3}$ These properties are highly expected for the large $N$ strongly coupled gauge theories. ${ }^{4}$ With this explicit identification of the spectrum of $C F T$ to the spectrum of the gravity on AdS space, we derived the GKPW relation.

In this paper, we include the $1 / N$ corrections to the study of [5], which are expected to correspond to the interactions in the gravity side, although we do not assume the existence of the gravity dual as in [5]. What we assume is the above two properties and the large $N$ factorization which is certainly satisfied for the t'Hooft large $N$ limit of gauge theories in the leading order in $N .^{5}$ Instead of including $1 / N$ corrections order by order, we will consider a large $N$ limit different form the large $N$ limit taken in [5] corresponding to free theory, which we will call the naive large $N$ limit below. This large $N$ limit considered in this paper corresponds to the classical limit of the theory, although there is no parameter like $\hbar$ in general a CFT, and the role of $\hbar$ is played by $1 / N^{2}$. In the gravity dual, the classical limit we consider in this paper, $1 / N^{2} \rightarrow 0$ is indeed realized by $G_{N} \rightarrow 0$ because $1 / G_{N}$ is an overall factor of the action and $1 / G_{N}$ will be identified as $N^{2}$.

More explicitly, we would like to understand what is the classical limit of the generic large $N$ gauge theories with conformal symmetry. In order to answer this, an important object is the algebra of the energy momentum tensor. This is an analogue of the Virasoro algebra for $d>2$. As conserved charges of the theory, an analogue of the Virasoro algebra is the algebra of the conformal symmetry generators. However, if we regard the generators of the Virasoro algebra as modes of the energy momentum tensor, we can consider the commutator algebra of the modes of the energy momentum tensor for $d>2$. Because of the assumptions, the generators of this algebra spans all operators on the low energy theories. Thus, the classical limit of the theory is given by the classical limit of this algebra, which gives the Poisson bracket. It is important to note that this algebra is almost unique, like Virasoro algebra, and the Brown-York tensor (or the boundary stress tensor) of classical gravity on asymptotic $A d S_{d+1}$ also forms the algebra by the Poisson bracket. We will show that the classical limit of the generic large $N$ gauge theory with conformal symmetry is the classical dynamics of the Einstein gravity on asymptotic $A d S_{d+1}$ by showing that the Poisson bracket and the Hamiltonian of the CFT are equivalent to the those of the gravity. This may be regarded as a kind of derivation of the AdS/CFT correspondence from CFT.

\footnotetext{
${ }^{2}$ This is similar to the hydrodynamics.

${ }^{3}$ Conversely, this complete independence is needed for the $C F T_{d}$ to have a gravity dual, in the limit.

${ }^{4}$ Here, the theories with weak t'Hooft coupling is not regarded as a generic theories.

${ }^{5}$ More explicitly, we will assume that only the energy momentum tensor is the low energy primary field which has large $C_{T}$ in (2.61) and the states generated by this are completely independent for the energy below some $C_{T}$-dependent large energy scale. This $C_{T}$ plays the role of $N^{2}$.
} 
Conversely, we can say that the classical dynamics of gravity on asymptotic $A d S_{d+1}$ is a classical dynamics of the energy momentum tensor of $C F T_{d}$. Thus, the quantization of gravity can be considered as finding a quantum system which has an "energy momentm tensor" which becomes the energy momentum tensor of CFT for a large central charge $C_{T}$ in the classical limit. (We also require that it has the generic spectrum in the large $C_{T}$ limit.) Of course, the natural choice for such theory is the CFT and the tensor as the energy momentum tensor itself. In general, the (classical) dynamics of an appropriate system in low energy limit will be described by the hydrodynamics in which only the energy momentum tensor appears. Because only the energy momentum tensor of the CFT appears in the classical gravity, this might mean that the classical gravity is a kind of thermal physics.

There are many things we do not understand in this paper. In particular, the black holes in the brick wall picture $[20,21]$ in the classical dynamics obtained in this paper will be interesting to be investigated. We hope to report this in near future.

This paper is organized as follows. In the next section, we study the algebra of the energy momentum tensor by expanding it on the cylinder. In section three, we consider the classical limit of the $C F T_{d}$ using generalized coherent states. It is shown that the classical limit of the $C F T_{d}$ is the classical dynamics of the Einstein gravity on asymptotic $A d S_{d+1}$ in the final section. In the appendix, relation between the classical limit of AdS/CFT and the naive large $N$ limit is explained and some discussions on generalized coherent states are given.

\section{Algebra for the energy momentum tensor}

In this section, we will expand the energy momentum tensor $T_{\mu \nu}$ of the $C F T_{d}$ on the cylinder $\mathbf{R} \times S^{d-1}$ by the "spherical harmonics" of $S^{d-1}$ and the energy, which is the eigen value of the dilatation $D$, to the infinitely many operators. These operators are analogues of the generators of the Virasoro algebra of the $C F T_{2}$. We will divide these operators to three classes (positive, negative and isotropy), which we will explain. Then, the commutator algebra of them will be studied from the operator product expansion (OPE). Because the low energy states are spanned by these operators acting on the vacuum, the algebra is considered as the operator algebra of the low energy theory of the generic large $N$ gauge theory with conformal symmetry.

For a review of the $C F T_{d}$, see, for example, [22].

\subsection{Scalar case}

First, as a warm-up example, let us consider the (normalized) scalar primary field $\mathcal{O}_{\Delta}(x)$, instead of $T_{\mu \nu}$, on $R^{d}$. Here, we regard the operators are defined by the radial quantization, thus, it is expanded by the spherical harmonics and the radial direction $|x|=\sqrt{\sum_{\mu=1}^{d} x^{\mu} x^{\mu}}$ as

$$
\mathcal{O}_{\Delta}(x)=\sum_{l=0}^{\infty} \sum_{m=0}^{m_{\max }(l)} Y_{l m}(\Omega) \mathcal{O}_{\Delta l m}(|x|),
$$

where $\mathcal{O}_{\Delta l m}(|x|)=\sum_{\omega}|x|^{\omega-\Delta} \mathcal{O}_{\Delta \omega l m}$ and $\mathcal{O}_{\Delta \omega l m}$ is the operator acting on the state of the theory on the $S^{d-1}$, whose coordinates are denoted by $\Omega$, and $\omega$ is the energy in the 
cylinder coordinates which is the eigen value of the dilatation $D$. The energy $\omega$ will take continuous values. Note that in the naive large $N$ limit taken in [5],

$$
n=(|\omega|-\Delta-l) / 2
$$

should be half integer because operators which violate this integer condition gives states which can not be in the states spanned by the descendant states of the primary operator $\mathcal{O}_{\Delta}(x)$. Including the $1 / N$ corrections, this $n$ will be slightly modified by the corrections. Moreover, the theory is not free theory except the naive large $N$ limit, $\omega$ can take any value which is the difference between the energies of the states of the theory. Here the spectrum of the states are almost the Fock space of the free theory, but it is modified by the $1 / N$ corrections. ${ }^{6}$ (Here, the conformal weight $\Delta$ is the one includes the $1 / N$ corrections.)

In (2.1), $m_{\max }(l)$ is the number of the independent spherical harmonics, which depends on $d$ and $l$. Here, this scalar field is assume to be Hermite. The scalar field on the cylinder, $d s^{2}=d \tau^{2}+d \Omega^{2}$ where $\tau=\log r$, is given by

$$
\mathcal{O}_{\Delta}^{c y}(\tau, \Omega)=\sum_{l=0}^{\infty} \sum_{m=0}^{m_{\max }(l)} Y_{l m}(\Omega) \sum_{\omega} e^{\omega \tau} \mathcal{O}_{\Delta \omega l m},
$$

then, the reflection positivity on the cylinder (or the usual Hermite conjugate on the Lorentzian cylinder) requires

$$
\mathcal{O}_{\Delta \omega l m}^{\dagger}=\mathcal{O}_{\Delta(-\omega) l m}
$$

where we take $Y_{l m}$ real.

Because $\mathcal{O}_{\Delta}(x)|0\rangle$ should be regular for $x=0$ and $Y_{l m}(\Omega)|x|^{l}$ is a polynomial of $x^{\mu}$, we see that $\mathcal{O}_{\Delta \omega l m}|0\rangle=0$ for $\omega<\Delta+l .{ }^{7}$ Similarly, the Hermite condition implies that $\langle 0| \mathcal{O}_{\Delta \omega l m}=0$ for $\omega>-(\Delta+l)$. Thus the operators $\mathcal{O}_{\Delta \omega l m}$ with $|\omega|<\Delta+l$ satisfy $0=\mathcal{O}_{\Delta \omega l m}|0\rangle=\langle 0| \mathcal{O}_{\Delta \omega l m}$ and we will denote these operators as $\mathcal{O}_{\Delta}^{\text {iso }}$. Note that the commutator of arbitrary two operators in $\mathcal{O}_{\Delta}^{\text {iso }}$ also satisfies $\left[\mathcal{O}_{\Delta \omega l m}, \mathcal{O}_{\Delta \omega^{\prime} l^{\prime} m^{\prime}}\right]|0\rangle=$ $\langle 0|\left[\mathcal{O}_{\Delta \omega l m}, \mathcal{O}_{\Delta \omega^{\prime} l^{\prime} m^{\prime}}\right]=0$. This $\mathcal{O}_{\Delta}^{\text {iso }}$ plays no roles in the naive large $N$ limit taken in [5] except the generators of the conformal group which are in $\mathcal{O}_{\Delta}^{\text {iso }}$ for the energy momentum tensor.

We will denote the operators $\mathcal{O}_{\Delta \omega l m}$ with $\omega \geq \Delta+l$ and $\omega \leq-(\Delta+l)$ as $\mathcal{O}_{\Delta}^{+}$and $\mathcal{O}_{\Delta}^{-}$, respectively. The operators in $\mathcal{O}_{\Delta}^{+}$correspond to the creation operators of the free scalar theory on the AdS background in the naive large $N$ limit [5], however, the notation used in this paper is slightly different from those in [5]. For this, we will explicitly explain the correspondence. First, for $\omega \geq \Delta+l$, we can see that

$$
\mathcal{O}_{\Delta \omega l m}=\frac{2^{-(2 n+l)}}{\int d \Omega} \frac{1}{n !} \frac{\Gamma\left(\frac{d}{2}\right)}{\Gamma\left(n+\frac{d}{2}+l\right)} s_{(l, m)}^{\mu_{1} \mu_{2} \ldots \mu_{l}} P_{\mu_{2}} \cdots P_{\mu_{l}}\left(P^{2}\right)^{n} \hat{\mathcal{O}}_{\Delta}
$$

\footnotetext{
${ }^{6}$ For the Virasoro algebra, $|\omega|=l$, which is an integer, because of the traceless condition and the current conservation, which is imposed by the e.o.m. There are no constraints like this for the higher dimensional case.

${ }^{7}$ We also see that if $n$ defined in (2.2) is not a half-integer, $\mathcal{O}_{\Delta \omega l m}|0\rangle=0$ by imposing the regularity of $\left(\partial_{x}^{2}\right)^{m} \mathcal{O}_{\Delta}(x)|0\rangle$ with a sufficiently large integer $m$.
} 
where $P^{\mu}$ act on an operator $\hat{\phi}$ such that $P^{\mu} \hat{\phi}=\left[\hat{P}^{\mu}, \hat{\phi}\right], \hat{\mathcal{O}}_{\Delta}=\left.\frac{1}{\int d \Omega} \mathcal{O}_{\Delta \omega l m}\right|_{\omega=\Delta, l=m=0}$ and $n=(\omega-\Delta-l) / 2$ should be a non-negative integer. Here, $s_{(l, m)}^{\mu_{1} \mu_{2} \ldots \mu_{l}}$ is a normalized rank $l$ symmetric traceless constant tensor which is related to the normalized spherical harmonics by

$$
Y_{l m}(\Omega)=|x|^{-l} s_{(l, m)}^{\mu_{1} \mu_{2} \ldots \mu_{l}} x_{\mu_{1}} x_{\mu_{2}} \cdots x_{\mu_{l}},
$$

where they are assumed to be normalized as

$$
\frac{1}{\int d \Omega} \int d \Omega Y_{l m}(\Omega) Y_{l^{\prime} m^{\prime}}(\Omega)=\delta_{l l^{\prime}} \delta_{m m^{\prime}}
$$

and $\int d \Omega=\frac{2(\pi)^{d / 2}}{\Gamma(d / 2)}$. To obtain this, we repeatedly use

$$
\left(\partial_{y}^{2}\right)\left(\left(y^{2}\right)^{n}\left(s_{(l, m)}^{\mu_{1} \mu_{2} \ldots \mu_{l}} y^{\mu_{1}} y^{\mu_{2}} \cdots y^{\mu_{l}}\right)\right)=4 n(n+l+d / 2-1)\left(y^{2}\right)^{n-1}\left(s_{(l, m)}^{\mu_{1} \mu_{2} \ldots \mu_{l}} y^{\mu_{1}} y^{\mu_{2}} \cdots y^{\mu_{l}}\right),
$$

and, for $l \leq l^{\prime}$,

$$
2^{l} \frac{\Gamma(l+d / 2)}{\Gamma(d / 2)} \delta_{l l^{\prime}} \delta_{m m^{\prime}}=\left(s_{\left(l^{\prime}, m^{\prime}\right)}^{\nu_{1} \nu_{2} \ldots \nu_{l^{\prime}}} \partial_{\nu_{1}} \partial_{\nu_{2}} \cdots \partial_{\nu_{l^{\prime}}}\right)\left(s_{(l, m)}^{\mu_{1} \mu_{2} \ldots \mu_{l}} x_{\mu_{1}} x_{\mu_{2}} \cdots x_{\mu_{l}}\right) .
$$

Then, the creation operator of the free theory in the naive large $N$ limit is given by

$$
\hat{a}_{n l m}^{\dagger}=\tilde{c}_{n l} \mathcal{O}_{\Delta \omega l m}
$$

where $c_{n l}$ is the normalization constant given by

$$
\tilde{c}_{n l}=\sqrt{\frac{\Gamma(\Delta)}{\Gamma(\Delta+n+l)} \frac{\Gamma\left(\Delta+1-\frac{d}{2}\right)}{\Gamma\left(\Delta+1-\frac{d}{2}+n\right)} n ! \frac{\Gamma\left(n+\frac{d}{2}+l\right)}{\Gamma\left(\frac{d}{2}\right)} \int d \Omega},
$$

which satisfies $\left[\hat{a}_{n l m}, \hat{a}_{n^{\prime} l^{\prime} m^{\prime}}^{\dagger}\right]=\delta_{n n^{\prime}} \delta_{l l^{\prime}} \delta_{m m^{\prime}}$ in the naive large $N$ limit.

\subsection{OPE and the commutator algebra}

In this subsection, we will consider the relation between the OPE and the commutator algebra. It is well known that the commutator algebra of the conserved charges are given by OPE using the deformation of the integration contour, like in $C F T_{2}$. However, this deformation technique can not be used for the operators which are not conserved charges, for example, the generic modes of the energy momentum tensor.

Here, we will explain how to derive, in principle, the algebra defined by the commutators of the operators $\mathcal{O}_{\Delta l m}(|x|)$ from the OPE of the corresponding primary field $\mathcal{O}_{\Delta}(x)$, which is assumed to be given as

$$
\mathcal{O}_{\Delta}(x) \mathcal{O}_{\Delta}(y)=\frac{1}{(x-y)^{2 \Delta}}+\cdots,
$$

where $\cdots$ includes the $1 / N$ suppressed terms which can be expanded by the primary fields [22] and (assuming the parity invariance) can be written as a sum of the following term:

$$
\frac{1}{(x-y)^{2 \Delta+l-\Delta^{\prime}}} u^{\mu_{1}} u^{\mu_{2}} \cdots u^{\mu_{l}} \mathcal{O}_{\mu_{1} \mu_{2}, \cdots \mu_{l}}^{\Delta^{\prime}}(y)
$$


where $u^{\mu}=x^{\mu}-y^{\mu}$ and $\mathcal{O}_{\mu_{1} \mu_{2}, \cdots \mu_{l}}^{\Delta^{\prime}}(y)$ is the (not necessary primary) fields with spin $l$ and conformal weight $\Delta^{\prime}$.

In the radial quantization, the commutator of the two fields at the equal "time" $|x|=1$ is given by

$$
\begin{aligned}
{\left[\mathcal{O}_{1}(x), \mathcal{O}_{2}(y)\right]_{|x|=|y|=1} } & =\lim _{\epsilon \rightarrow 0}\left(\left.\left.\mathcal{O}_{1}(x)\right|_{|x|=1+\epsilon} \mathcal{O}_{2}(y)\right|_{|y|=1}-\left.\left.\mathcal{O}_{2}(y)\right|_{|y|=1} \mathcal{O}_{1}(x)\right|_{|x|=1-\epsilon}\right), \quad, 2.14 \\
& =\lim _{\epsilon \rightarrow 0}\left(\left.\left(\mathcal{O}_{1}(x) \mathcal{O}_{2}(y)\right)\right|_{|x|=1+\epsilon,|y|=1}-\left.\left(\mathcal{O}_{2}(y) \mathcal{O}_{1}(x)\right)\right|_{|x|=1-\epsilon,|y|=1}\right),
\end{aligned}
$$

where

$$
\mathcal{O}_{1}(x)=\frac{\partial^{p}}{\partial^{p}|x|} \mathcal{O}_{\Delta}(x), \quad \mathcal{O}_{2}(y)=\frac{\partial^{q}}{\partial^{q}|y|} \mathcal{O}_{\Delta}(y) .
$$

We will use the OPE expansion for evaluating the r.h.s. of (2.14). One might worry about the other operator insertions near $x$ or $y$ which may invalidate the OPE expansion. We will explain below this is not the case for the evaluation of the commutator. Note that the equal time commutator of the local fields should vanish if $x \neq y$ because of the causality in the Lorentzian cylinder. Thus, for non-vanishing commutator, $\left(\mathcal{O}_{1}(x) \mathcal{O}_{2}(y)\right)_{|x|=1 \pm \epsilon,|y|=1}$ in $(2.14)$ can be given by evaluating $\frac{\partial^{p}}{\partial^{p}|x|} \frac{\partial^{q}}{\partial^{q}|y|}\left(\mathcal{O}_{\Delta}(x) \mathcal{O}_{\Delta}(y)\right)$ with the OPE for $\mathcal{O}_{\Delta}(x) \mathcal{O}_{\Delta}(y)$ because $x=y$ and any other operator insertions are not close. For $x \neq y$, the OPE is valid if there are no operator insertions near $x$ or $y$ and for this case the commutator should be zero using the OPE for the r.h.s. of (2.14). However, even if there are operator insertions, the commutator should be zero because of the causality, although we can not use the OPE expansion. Therefore, we can use the OPE for evaluating the r.h.s. of (2.14) for any $x, y$.

Furthermore, only the singular parts of the OPE can contribute the commutator because "regular" terms vanishes if we take a limit $x \rightarrow y$. (Here, the "regular" terms mean terms which vanish in the limit. However, the terms with non-integer conformal weight will become singular term by acting $\frac{\partial^{p}}{\partial^{p}|x|}$ with sufficiently large $p$.)

Because the commutator (2.14) will contain a delta function for the "space" directions $\Omega$ as usual, it will be convenient to consider the commutator of the modes of fields which are decomposed by the spherical Harmonics of "space" directions:

$$
\begin{aligned}
& {\left[\frac{\partial^{p}}{\partial^{p}|x|} \mathcal{O}_{\Delta l m}(|x|), \frac{\partial^{q}}{\partial^{q}|y|} \mathcal{O}_{\Delta l m}(|y|)\right]_{|x|=|y|=1}} \\
& =\frac{1}{\left(\int d \Omega\right)^{2}} \int d \Omega Y_{l m}(\Omega) \int d \Omega^{\prime} Y_{l^{\prime} m^{\prime}}^{\prime}\left(\Omega^{\prime}\right)\left[\mathcal{O}_{1}(x), \mathcal{O}_{2}(y)\right]_{|x|=|y|=1}
\end{aligned}
$$

where $y$ also are decomposed to the radial direction $|y|$ and the angular directions $\Omega^{\prime}$ for $S^{d-1}$.

We will first concentrate on the large $N$ leading term in the OPE (2.12). Then, we have the expansion of the correlation function as

$$
\frac{1}{(x-y)^{2 \Delta}}=\frac{1}{\left(r_{>}\right)^{2 \Delta}} \frac{1}{\left(1+\left(\frac{r_{<}}{r_{>}}\right)^{2}-2\left(\frac{r_{<}}{r_{>}}\right) \eta\right)^{\Delta}}=\frac{1}{\left(r_{>}\right)^{2 \Delta}} \sum_{s=0}^{\infty}\left(\frac{r_{<}}{r_{>}}\right)^{s} C_{s}^{\Delta}(\eta),
$$


where $\eta=\frac{x^{\mu} y_{\mu}}{|x||y|}$

$$
C_{s}^{\Delta}(\eta)=\sum_{p=0}^{\left[\frac{1}{2} s\right]} \frac{(-1)^{p}(2 \eta)^{s-2 p}}{p !(s-2 p) !} \frac{\Gamma(\Delta+s-p)}{\Gamma(\Delta)}
$$

is the Gegenbauer polynomial [23] which reduces to the Legendre polynomial for $\Delta=1 / 2$ and $r_{>}$and $r_{<}$are the larger and smaller ones of $|x|$ and $|y|$, respectively. By the rotational invariance, we can write

$$
C_{s}^{\Delta}(\eta)=\sum_{n=0}^{\left[\frac{1}{2} s\right]}\left(d^{\Delta}\right)_{s}^{s-2 n} \sum_{m} Y_{(s-2 n) m}^{*}(\Omega) Y_{(s-2 n) m}\left(\Omega^{\prime}\right),
$$

where $\Omega$ and $\Omega^{\prime}$ are the angular variables of $x$ and $y$, respectively, and $\left(d^{\Delta}\right)_{s}^{l}=0$ for $l>s$ or $s-l$ is odd. For $\Delta=d / 2-1$, we can see $\left(d^{\Delta}\right)_{s}^{l}=\frac{(d-2) 2 \pi^{\frac{d}{2}}}{(d+2 s-2) \Gamma(d / 2)} \delta_{s l}$ [23]. This implies the relations between $\sum Y^{*}(\Omega) Y\left(\Omega^{\prime}\right)$ and $(2 \eta)^{s-2 p}$ :

$$
\frac{(d-2) 2 \pi^{\frac{d}{2}}}{(d+2 s-2) \Gamma(d / 2)} \sum_{m} Y_{(s-2 n) m}^{*}(\Omega) Y_{(s-2 n) m}\left(\Omega^{\prime}\right)=\sum_{p=0}^{\left[\frac{1}{2} s\right]} \frac{(-1)^{p}(2 \eta)^{s-2 p}}{p !(s-2 p) !} \frac{\Gamma(d / 2-1+s-p)}{\Gamma(d / 2-1)} .
$$

Using these relations, we find

$$
\left(d^{\Delta}\right)_{s}^{s-2 n}=\frac{2 \pi^{\frac{d}{2}}}{\Gamma(d / 2)} \frac{\Gamma(\Delta+s-n)}{\Gamma(\Delta)} \frac{\Gamma\left(\Delta+1-\frac{d}{2}+n\right)}{\Gamma\left(\Delta+1-\frac{d}{2}\right)} \frac{1}{n !} \frac{\Gamma\left(\frac{d}{2}\right)}{\Gamma\left(s-n+\frac{d}{2}\right)},
$$

and the correlator is expressed as

$$
\frac{1}{(x-y)^{2 \Delta}}=\frac{1}{\left(r_{>}\right)^{2 \Delta}} \sum_{s=0}^{\infty}\left(\frac{r_{<}}{r_{>}}\right)^{s} \sum_{n=0}^{\left[\frac{1}{2} s\right]}\left(d^{\Delta}\right)_{s}^{s-2 n} \sum_{m} Y_{(s-2 n) m}^{*}(\Omega) Y_{(s-2 n) m}\left(\Omega^{\prime}\right) .
$$

Thus, in the naive large $N$ limit, the equal time commutator between the modes is given by

$$
\begin{aligned}
& {\left[\frac{\partial^{p}}{\partial^{p}|x|} \mathcal{O}_{\Delta l m}(|x|), \frac{\partial^{q}}{\partial^{q}|y|} \mathcal{O}_{\Delta l m}(|y|)\right]_{|x|=|y|=1}=\frac{1}{\left(\int d \Omega\right)^{2}} \sum_{s=0}^{l} \delta_{l l^{\prime}} \delta_{m m^{\prime}}\left(d^{\Delta}\right)_{s}^{l}} \\
& \quad \times\left(\left(\frac{\partial^{p}}{\partial^{p} r} r^{-s-2 \Delta}\right)_{r=1}\left(\frac{\partial^{q}}{\partial^{q} r} r^{s}\right)_{r=1}-\left(\frac{\partial^{q}}{\partial^{q} r} r^{-s-2 \Delta}\right)_{r=1}\left(\frac{\partial^{p}}{\partial^{p} r} r^{s}\right)_{r=1}\right),
\end{aligned}
$$

where we have used

$$
\begin{gathered}
\lim _{\epsilon \rightarrow 0} \int d \Omega Y_{l m}(\Omega) \int d \Omega^{\prime} Y_{l^{\prime} m^{\prime}}^{\prime}\left(\Omega^{\prime}\right)\left[\frac{\partial^{p}}{\partial^{p}|x|} \frac{\partial^{q}}{\partial^{q}|y|}\left(\frac{1}{(x-y)^{2 \Delta}}\right)\right]_{|x|=1+\epsilon,|y|=1} \\
=\sum_{s=0}^{l} \delta_{l l^{\prime}} \delta_{m m^{\prime}}\left(d^{\Delta}\right)_{s}^{l}\left(\frac{\partial^{p}}{\partial^{p} r} r^{-s-2 \Delta}\right)_{r=1}\left(\frac{\partial^{q}}{\partial^{q} r} r^{s}\right)_{r=1},
\end{gathered}
$$

and similar one for $|x|=1-\epsilon$. Note that the two different expansions, which originate from the different limits of the integration contours, $(|x|=1+\epsilon$ and $|x|=1-\epsilon)$, of 
the same function give the different results which make the commutator non-vanishing. Note also that the commutator for the modes is indeed finite because there are no infinite summations.

If we know the spectrum, we can also find the modes with fixed energy by the expansion: $\mathcal{O}_{\Delta l m}(|x|)=\sum_{\omega}|x|^{\omega-\Delta} \mathcal{O}_{\Delta \omega l m}$. Then, the commutator of the operators $\mathcal{O}_{\Delta \omega l m}$ should satisfy (2.23). Comparing the exponent of $|x|,|y|$, a simple solution is

$$
\begin{aligned}
{\left[\mathcal{O}_{\Delta \omega l m}, \mathcal{O}_{\Delta \omega^{\prime} l m}\right] } & =\frac{1}{\left(\int d \Omega\right)^{2}} \sum_{s=0}^{l} \delta_{l l^{\prime}} \delta_{m m^{\prime}}\left(d^{\Delta}\right)_{s}^{l}\left(\delta_{-s-2 \Delta \omega-\Delta} \delta_{s \omega^{\prime}-\Delta}-\delta_{-s-2 \Delta \omega^{\prime}-\Delta} \delta_{s \omega-\Delta}\right) \\
& =\frac{1}{\left(\int d \Omega\right)^{2}} \delta_{\omega+\omega^{\prime}} \delta_{l l^{\prime}} \delta_{m m^{\prime}}\left(\left(d^{\Delta}\right)_{-\Delta+\omega^{\prime}}^{l}-\left(d^{\Delta}\right)_{-\Delta+\omega}^{l}\right) \\
& =\frac{1}{\left(\int d \Omega\right)^{2}} \delta_{\omega+\omega^{\prime}} \delta_{l l^{\prime}} \delta_{m m^{\prime} \operatorname{sgn}}\left(\omega^{\prime}\right)\left(d^{\Delta}\right)_{\left|\omega^{\prime}\right|-\Delta}^{l},
\end{aligned}
$$

which agrees with (2.10).

Let us consider the full OPE, not only the large $N$ leading term, which includes terms written as

$$
\frac{1}{(x-y)^{2 \Delta+l-\Delta^{\prime}}} u^{\mu_{1}} u^{\mu_{2}} \cdots u^{\mu_{l}} \mathcal{O}_{\mu_{1} \mu_{2}, \cdots \mu_{l}}^{\Delta^{\prime}}(y) \text {. }
$$

Then, the $\frac{1}{(x-y)^{2 \Delta+l-\Delta^{\prime}}}$ factor can be expanded by $(2.22)$ and $\mathcal{O}_{\mu_{1} \mu_{2}, \cdots \mu_{l}}^{\Delta^{\prime}}(y)$ also can be expanded by the spherical functions and the symmetric tensor harmonics for $d>3$. In order to compute the commutator, as for the leading order, we just need to decompose the products of irreducible representations of $\mathrm{SO}(d)$. For $d=3$ the Clebsh-Gordon coefficients are well known and for other $d$ we can compute it, order by order, at least. Thus, in principle, we can compute the commutator and it contains only a finite number of terms as for the large $N$ leading order. It should be noted that the terms (2.26) which is nondivergent at $x-y=0$, do not contribute to the commutator because the two expansions are same. Therefore, the commutators of the modes, $\left[\frac{\partial^{p}}{\partial^{p}|x|} \mathcal{O}_{\Delta l m}(|x|), \frac{\partial^{q}}{\partial^{q}|y|} \mathcal{O}_{\Delta l m}(|y|)\right]_{|x|=|y|=1}$ ' can be determined by the singular terms of the OPE, which satisfies $2 \Delta-\Delta^{\prime}>0$ if $2 \Delta-\Delta^{\prime}$ is integer, or $2 \Delta-\Delta^{\prime}+p>0$ if $2 \Delta-\Delta^{\prime}$ is not integer.

For the modes with fixed energy, the commutator of the operators $\mathcal{O}_{\Delta \omega l m}$ should satisfy (2.16) and comparing the exponent of $|x|,|y|$, a simple solution for the commutator of the operators with a general OPE is

$$
\begin{aligned}
{\left[\mathcal{O}_{\Delta \omega l m}, \mathcal{O}_{\Delta \omega^{\prime} l^{\prime} m^{\prime}}\right]=} & \int d \Omega Y_{l m}(\Omega) \int d \Omega^{\prime} Y_{l^{\prime} m^{\prime}}^{\prime}\left(\Omega^{\prime}\right) \\
& \times\left(\mathrm{P}_{+}\left[|x|^{\Delta-\omega}|y|^{\Delta-\omega^{\prime}} \mathcal{O}_{\Delta}(x) \mathcal{O}_{\Delta}(y)\right]-\mathrm{P}_{-}\left[|x|^{\Delta-\omega}|y|^{\Delta-\omega^{\prime}} \mathcal{O}_{\Delta}(x) \mathcal{O}_{\Delta}(y)\right]\right)
\end{aligned}
$$

where $\mathrm{P}_{ \pm}[f(x, y)]$ is defined as follows: let us consider a function $f(x, y)$ which has an expansion of the form as $f(x, y)=|x|^{a}|y|^{-b} \sum_{m=0}^{\infty}(|y| /|x|)^{m} f_{m}\left(\Omega, \Omega^{\prime}\right)$ for $|x|>|y|$. Then, $\mathrm{P}_{+}[f(x, y)]$ is the zero-mode of this expansion, i.e. $\mathrm{P}_{+}[f(x, y)]=f_{a}\left(\Omega, \Omega^{\prime}\right)$ if $a=b$ and $a$ is non-negative integer, otherwise $\mathrm{P}_{+}[f(x, y)]=0$. On the other hand, for the function $f(x, y)$ which is written as $f(x, y)=|y|^{a^{\prime}}|x|^{-b^{\prime}} \sum_{m=0}^{\infty}(|x| /|y|)^{m} f_{m}\left(\Omega, \Omega^{\prime}\right)$ for $|y|>|x|$, 
$\mathrm{P}_{-}[f(x, y)]=f_{a}\left(\Omega, \Omega^{\prime}\right)$ if $a^{\prime}=b^{\prime}$ and $a^{\prime}$ is non-negative integer, otherwise $\mathrm{P}_{-}[f(x, y)]=0$. Note that the $\mathrm{OPE}$ of $\mathcal{O}_{\Delta}(x) \mathcal{O}_{\Delta}(y)$ has such expansions depending on the sign of $|x|-|y|$. We can check this formula for the leading order case. With these expansion formulas, we can evaluate (2.27) as

$$
\begin{aligned}
\int d \Omega Y_{l m}(\Omega) \int d \Omega^{\prime} Y_{l^{\prime} m^{\prime}}^{\prime}\left(\Omega^{\prime}\right) \mathrm{P}_{+}\left[|x|^{\Delta-\omega}|y|^{\Delta-\omega^{\prime}} \frac{1}{(x-y)^{2 \Delta}}\right] & =\sum_{q=0}^{\infty} \delta_{\Delta+\omega+q} \delta_{\Delta-\omega^{\prime}+q} \delta_{l l^{\prime}} \delta_{m m^{\prime}}\left(d^{\Delta}\right)_{q}^{l} \\
& =\delta_{\omega+\omega^{\prime}} \delta_{l l^{\prime}} \delta_{m m^{\prime}}\left(d^{\Delta}\right)_{-\Delta+\omega^{\prime}}^{l},
\end{aligned}
$$

which can be non-zero for $\Delta \leq \omega^{\prime} \leq \Delta+l$, and

$$
\begin{aligned}
\int d \Omega Y_{l m}(\Omega) \int d \Omega^{\prime} Y_{l^{\prime} m^{\prime}}^{\prime}\left(\Omega^{\prime}\right) \mathrm{P}_{-}\left[|x|^{\Delta-\omega}|y|^{\Delta-\omega^{\prime}} \frac{1}{(x-y)^{2 \Delta}}\right] & =\sum_{q=0}^{\infty} \delta_{\Delta+\omega^{\prime}+q} \delta_{\Delta-\omega+q} \delta_{l l^{\prime}} \delta_{m m^{\prime}}\left(d^{\Delta}\right)_{q}^{l} \\
& =\delta_{\omega+\omega^{\prime}} \delta_{l l^{\prime}} \delta_{m m^{\prime}}\left(d^{\Delta}\right)_{-\Delta+\omega}^{l}, \quad(2.29)
\end{aligned}
$$

which can be non-zero for $\Delta \leq \omega \leq \Delta+l$. Thus, the commutators is given by

$$
\begin{aligned}
{\left[\mathcal{O}_{\Delta \omega l m}, \mathcal{O}_{\Delta \omega^{\prime} l^{\prime} m^{\prime}}\right] } & =\delta_{\omega+\omega^{\prime}} \delta_{l l^{\prime}} \delta_{m m^{\prime}}\left(\left(d^{\Delta}\right)_{-\Delta+\omega^{\prime}}^{l}-\left(d^{\Delta}\right)_{-\Delta+\omega}^{l}\right)+\cdots \\
& =\delta_{\omega+\omega^{\prime}} \delta_{l l^{\prime}} \delta_{m m^{\prime}} \operatorname{sgn}\left(\omega^{\prime}\right)\left(d^{\Delta}\right)_{\left|\omega^{\prime}\right|-\Delta}^{l}+\cdots
\end{aligned}
$$

which indeed coincides with (2.25).

In this subsection, we have seen that with the singular parts of the OPE, the commutator of the operators are given by (2.16) or (2.27) using the expansion (2.17), at least, in principle. This will be used below.

\subsection{Algebra for the energy momentum tensor}

As for the scalar, we will consider the commutator algebra for the energy momentum tensor.

We will expand the energy momentum tensor with the traceless and conserved properties. First, we will use the coordinates $r=|x|$ and $z^{i}$ as the coordinates of $S^{d-1}$, where $(i=1, \ldots, d-1)$, with the flat metric $d s^{2}=d r^{2}+r^{2} g_{i j}^{S^{d-1}} d z^{i} d z^{j}$. We decompose it as

$$
T_{\mu \nu}(x)=\frac{\partial z^{i}}{\partial x^{\mu}} \frac{\partial z^{j}}{\partial x^{\nu}} T_{i j}(x)+\frac{x^{\mu}}{r} \frac{\partial z^{j}}{\partial x^{\nu}} T_{r j}(x)+\frac{x^{\nu}}{r} \frac{\partial z^{i}}{\partial x^{\mu}} T_{i r}(x)+\frac{x^{\mu}}{r} \frac{x^{\nu}}{r} T_{r r}(x),
$$

where

$$
\begin{aligned}
& T_{i j}(x)=e_{i}^{\mu} e_{j}^{\nu} T_{\mu \nu}(x), \\
& T_{r j}(x)=\frac{x^{\mu}}{r} e_{j}^{\nu} T_{\mu \nu}(x), \\
& T_{r r}(x)=\frac{x^{\mu}}{r} \frac{x_{\nu}}{r} T_{\mu \nu}(x),
\end{aligned}
$$


and $e_{i}^{\mu}=\frac{\partial x^{\mu}}{\partial z^{i}} e_{r}^{\mu}=\frac{\partial x^{\mu}}{\partial r}=\frac{x^{\mu}}{r}$. Then, for the $S^{d-1}$ directions, we will uniquely decompose them as

$$
\begin{gathered}
T_{i j}\left(r, z^{i}\right)=\sum_{\omega} r^{\omega-d+2}\left(\sum_{l=2} \sum_{m} t_{\omega l m}^{T} Y_{i j}^{l m}\left(z^{i}\right)+\sum_{l=2, m} t_{\omega l m}^{V}\left(D_{i} Y_{j}^{l m}\left(z^{i}\right)+D_{j} Y_{i}^{l m}\left(z^{i}\right)\right)\right. \\
\left.+\sum_{l=2} \sum_{m} t_{\omega l m}^{S}\left(D_{i} D_{j}-\frac{1}{d-1} g_{i j}^{S^{d-1}} D_{k} D_{k}\right) Y^{l m}\left(z^{i}\right)+\sum_{l=0} \sum_{m} t_{\omega l m}^{t r a c e} \frac{1}{d-1} g_{i j}^{S^{d-1}} Y^{l m}\left(z^{i}\right)\right) \\
T_{r i}\left(r, z^{i}\right)=\sum_{\omega} r^{\omega-d+1}\left(\sum_{l=1} \sum_{m} v_{\omega l m}^{V} Y_{i}^{l m}\left(z^{i}\right)+\sum_{l=1} \sum_{m} v_{\omega l m}^{S} D_{i} Y^{l m}\left(z^{i}\right)\right)
\end{gathered}
$$

and

$$
T_{r r}\left(r, z^{i}\right)=\sum_{\omega} r^{\omega-d} \sum_{l=0} \sum_{m} s_{\omega l m}^{S} Y^{l m}\left(z^{i}\right)
$$

Here, the rank $r$ symmetric (traceless) tensor harmonics on unit radius $S^{d-1}, Y_{i_{1}, i_{2}, \cdots, i_{r}}^{l m}(\Omega)$, is defined such that $Y_{i_{1}, i_{2}, \cdots, i_{r}}^{l m}$ is totally symmetric for the indices $i_{k}$ and

$$
\begin{aligned}
D^{i} D_{i} Y_{j_{1}, j_{2}, \cdots, j_{r}}^{l m} & =(-l(l+d-2)+r) Y_{j_{1}, j_{2}, \cdots, j_{r}}^{l m}, \\
D^{i} Y_{i, i_{2}, \cdots, i_{r}}^{l m} & =0 \\
g_{S^{d-1}}^{i j} Y_{i, j, i_{3} \cdots, i_{r}}^{l m} & =0
\end{aligned}
$$

where $z^{i}(i=1,2, \cdots, d-1)$ are coordinate of $S^{d-1}, D_{i}$ is the covariant derivative on $S^{d-1}$, and $g_{S^{d-1}}^{i j}$ is the inverse metric of unit radius $S^{d-1}$. Here, $l=r, r+1, r+2, \cdots$ and $m$ runs from 1 to the number of the independent harmonics which depends on $l$ and $r$. This harmon$\operatorname{ics} Y_{i_{1}, i_{2}, \cdots, i_{r}}^{(r) l m}$ is the unitary representation of $\mathrm{SO}(d)$ which corresponds to the Young diagram labeled by $[l, r, 0, \ldots, 0]$. More details for the symmetric tensor harmonics, see [27-30].

The energy $\omega$ should be an integer in the naive large $N$ limit because there are no states with non-integer energy for the primary field with the integer conformal weight. This implies that a possible energy $\omega$ is an integer except $1 / N^{2}$ corrections, which is expected from the usual large $N$ expansion. Note that the modes with fixed energy, for example $t_{\omega l m}^{T}$, may be identically zero depending on $\omega$.

We will show that the rank $r$ symmetric (traceless) tensor harmonics can be represented by

$$
Y_{j_{1}, j_{2}, \cdots, j_{r}}^{l m}=s_{\mu_{1} \mu_{2} \cdots \mu_{r} ; \nu_{1} \nu_{2} \cdots \nu_{l}}^{r l m} \frac{e_{j_{1}}^{\mu_{1}}}{r} \frac{e_{j_{2}}^{\mu_{1}}}{r} \cdots \frac{e_{j_{r}}^{\mu_{1}}}{r} \frac{x^{\nu_{1}}}{r} \frac{x^{\nu_{2}}}{r} \cdots \frac{x^{\nu_{l}}}{r}
$$

where $l \geq r$ and $s_{\mu_{1} \mu_{2} \cdots \mu_{r} ; \nu_{1} \nu_{2} \cdots \nu_{l}}^{r l m}$ is a traceless constant tensor which is given by the antisymmetrization of the $r$ pairs of the following indices: $\left(\mu_{1}, \nu_{1}\right),\left(\mu_{2}, \nu_{2}\right), \cdots,\left(\mu_{r}, \nu_{r}\right)$, and then the symmetrization for the $\mu_{a}$ and $\nu_{a}$. This (anti)symmetrization procedure corresponds to the Young diagram labeled by $[l, r, 0, \ldots, 0]$. Using

$$
\delta_{\nu}^{\mu}=\partial_{\rho} x^{\mu} \partial^{\rho} x^{\nu}=\nabla_{i} x^{\mu} \nabla^{i} x^{\nu}+\nabla_{r} x^{\mu} \nabla^{r} x^{\nu}=\frac{1}{r^{2}} g_{S^{d-1}}^{i j} e_{i}^{\mu} e_{j}^{\nu}+\frac{1}{r^{2}} x^{\mu} x^{\nu}
$$


where $\nabla$ is the covariant derivative on $\mathbf{R}^{d}$, we can see that $g_{S^{d-1}}^{i j} Y_{i, j, i_{3} \cdots, i_{r}}^{l m}=0$ because $s_{\mu_{1} \mu_{2} \cdots \mu_{r} ; \nu_{1} \nu_{2} \cdots \nu_{l-r}}^{r l m}$ is traceless and anti-symmetric for the interchanging a pair of $\mu$ and $\nu$. Next, note that the Christoffel symbols in the coordinates $\left\{z^{i}, r\right\}$ of $\mathbf{R}^{d}$ are given by

$$
\Gamma_{j r}^{i}=\frac{1}{r} \delta_{j}^{i}=\Gamma_{r j}^{i}, \quad \Gamma_{i j}^{r}=-r g_{i j}^{S^{d-1}}, \quad \Gamma_{i j}^{k}=\Gamma_{i j}^{S^{d-1} k},
$$

where $\Gamma^{S^{d-1} k}$ is the Christoffel symbols of the $S^{d-1}$, and others vanish. Then, we can show that

$$
0=e_{i}^{\alpha} e_{j}^{\beta} \nabla_{\alpha} \nabla_{\beta} x^{\mu}=\nabla_{i} \nabla_{j} x^{\mu}=D_{i} e_{j}^{\mu}+g_{i j}^{S^{d-1}} x^{\mu},
$$

which implies $D^{i} e_{i}^{\mu}=-(d-1) x^{\mu}, D^{i} D_{i} e_{j}^{\mu}=-e_{j}^{\mu}$ and $g_{S^{d-1}}^{j k} e_{j}^{\nu} D_{i} e_{k}^{\mu}=-e_{i}^{\nu} x^{\mu}$. Using these, we can see that $D^{i} Y_{i, i_{2}, \cdots, i_{r}}^{l m}=0$ and $D^{i} D_{i} Y_{j_{1}, j_{2}, \cdots, j_{r}}^{l m}=(-l(l+d-2)+r) Y_{j_{1}, j_{2}, \cdots, j_{r}}^{l m}$ hold because of the traceless and (anti)symmetric properties of $s^{n l m}$.

In the expansion (2.33), the traceless condition of the energy momentum tensor is just

$$
t_{\omega l m}^{\text {trace }}=-s_{\omega l m}^{S} .
$$

For the conservation condition $\partial^{\mu} T_{\mu \nu}=0$, we will use following formula:

$$
\begin{aligned}
\partial^{\mu} T_{\mu r} & =\left(\frac{\partial}{\partial r}+\frac{d}{r}\right) T_{r r}+\frac{1}{r^{2}} D^{i} T_{i r}, \\
\partial^{\mu} T_{\mu i} & =\left(\frac{\partial}{\partial r}+\frac{d-1}{r}\right) T_{r i}+\frac{1}{r^{2}} D^{j} T_{j i} .
\end{aligned}
$$

With these, we find, for $l \geq 2$,

$$
\begin{aligned}
\omega s_{\omega l m}^{S} & =l(l+d-2) v_{\omega l m}^{S}, \quad \omega v_{\omega l m}^{V}=(l+d-1)(l-1) t_{\omega l m}^{V} \\
s_{\omega l m}^{S} & =\omega(d-1) v_{\omega l m}^{S}-(d-2)(l+d-1)(l-1) t_{\omega l m}^{S},
\end{aligned}
$$

where we have used $\left(D_{i} D_{j}-D_{j} D_{i}\right) V_{k}=V_{l} R_{i k j}^{l}$ and $R_{i j k l}=g_{i l} g_{j k}-g_{i k} g_{j l}$ for unit radius sphere. Thus, for $l \geq 2$, only the $s_{\omega l m}^{S}, v_{\omega l m}^{V}, t_{\omega l m}^{T}$ are the independent operators for the energy momentum tensor. We find, for $l=1$,

$$
\omega v_{\omega l m}^{V}=0, \quad \omega s_{\omega l m}^{S}=(d-1) v_{\omega l m}^{S}, \quad s_{\omega l m}^{S}=\omega(d-1) v_{\omega l m}^{S},
$$

with $t_{\omega l m}^{S}=t_{\omega l m}^{V}=t_{\omega l m}^{T}=0$, and, for $l=0$,

$$
\omega s_{\omega l m}^{S}=0
$$

with $t_{\omega l m}^{S}=t_{\omega l m}^{V}=t_{\omega l m}^{T}=v_{\omega l m}^{V}=v_{\omega l m}^{S}=0$. Thus, the non-trivial operators for $l=0,1$ are $v_{01 m}^{V}, s_{ \pm 11 m}^{S}=v_{ \pm 11 m}^{S}$ and $s_{000}^{S}$. All of these correspond to the generators of the conformal symmetry. Indeed, inserting the expansion of the $T_{\mu \nu}(x)$ into the definition of the generators,

$$
Q_{\epsilon}\left(S^{d-1}\right)=-\int_{S^{d-1}} d S_{\mu} \epsilon_{\nu} T^{\mu \nu}(x)
$$

where $\epsilon_{\nu}$ is the conformal Killing vector and integration is on $S^{d-1}$ at a fixed $r$, we find that $D \sim s_{000}^{S}, M_{\mu \nu} \sim s_{\mu \nu}^{11 m} v_{01 m}^{V}, P^{\mu} \sim s_{\mu}^{01 m} s_{11 m}^{S}, K^{\mu} \sim s_{\mu}^{01 m} s_{-11 m}^{S}$ for any choice of $r$ as 
required from the conservation law. Note that all the generators of the conformal symmetry have $l=0,1$ in our notation.

We will denote a set of the non-trivial $s_{\omega l m}^{S}, v_{\omega l m}^{V}, t_{\omega l m}^{T}$ as

$$
L_{A \omega l m} \equiv\left\{s_{\omega l m}^{S}, v_{\omega l m}^{V}, t_{\omega l m}^{T}\right\},
$$

where the index $A$ takes $S, V, T$. We define also the mode of the energy momentum tensor decomposed for the space direction $\Omega$ as

$$
L_{A l m}(|x|)=\sum_{\omega} r^{\omega-d-r[A]+2} L_{A \omega l m} .
$$

Let us consider the spectrum of the low energy theory. The energy momentum tensor is Hermite on the cylinder, then,

$$
L_{A \omega l m}^{\dagger}=L_{A(-\omega) l m},
$$

where we have taken $Y_{i_{1}, i_{2}, \cdots, i_{r}}^{l m}$ real. Here, the energy momentum tensor on the cylinder $\left(r=e^{\tau}\right)$ is given by

$$
T_{i j}^{c y}\left(\tau, z^{i}\right)=r^{d-2} T_{i j}\left(r, z^{i}\right), T_{\tau i}^{c y}\left(\tau, z^{i}\right)=r^{d-1} T_{r i}\left(r, z^{i}\right), T_{\tau i}^{c y}(\tau, \tau)=r^{d} T_{r r}\left(r, z^{i}\right),
$$

where extra $1 / r$ factors are from the normalizing $d x^{\mu} / d z^{i}{ }^{8}$ This energy momentum tensor indeed satisfies the conservation law.

As for the scalar case, we will require the regularity of $T_{\mu \nu}(x)|0\rangle$ at $r=0$. For $L_{T \omega l m}$, this means $L_{T \omega l m}|0\rangle=0$ for $\omega<d+l$ because $\frac{\partial z^{i}}{\partial x^{\mu}} \frac{\partial z^{j}}{\partial x^{\nu}} Y_{i j}^{l m}\left(z^{i}\right)=$ $s_{\mu_{1} \mu_{2} ; \nu_{1} \nu_{2} \cdots \nu_{l}}^{r l m} \delta_{\mu}^{\mu_{1}} \delta_{\nu}^{\mu_{2}} \frac{1}{r^{2}} \frac{x^{\nu_{1}}}{r} \frac{x^{\nu_{2}}}{r} \cdots \frac{x^{\nu} l}{r}$. Similarly, we can see that

$$
L_{A \omega l m}|0\rangle=0 \text { for } \omega<d+l+r[A]-2,
$$

where $r[A]=0,1,2$ for $A=S, V, T$, respectively. This can be checked by considering $T_{\mu \nu}(x)=c_{\mu \nu}$, which is constant and traceless. This is the lowest regular term and in the polar coordinates, $T_{i j}(x)=e_{i}^{\mu} e_{j}^{\nu} c_{\mu \nu}, T_{r j}(x)=\frac{x^{\mu}}{r} e_{j}^{\nu} c_{\mu \nu}, T_{r r}(x)=\frac{x^{\mu}}{r} \frac{x_{\nu}}{r} c_{\mu \nu}$. This corresponds to $s_{\omega l m}^{S}$ with $\omega=d, l=2$, which is the boundary of the condition (2.54). For $L_{V \omega l m}$, we can check (2.54) by considering $T_{\mu \nu}(x)=c_{\mu \nu \rho} x^{\rho}$.

The Hermite condition implies that $\langle 0| L_{A \omega l m}=0$ for $\omega>-(d+l+r[A]-2)$. Thus the operators $L_{A \omega l m}$ with $|\omega|<d+l+r[A]-2$ satisfy $0=L_{A \omega l m}|0\rangle=\langle 0| L_{A \omega l m}$ and we will denote these operators as $L^{\text {iso }}$, which includes the generators of the conformal group. We will also denote the operators $L_{A \omega l m}$ with $\omega \geq d+l+r[A]-2$ and $\omega \leq-(d+l+r[A]-2)$ as $L^{+}$and $L^{-}$, respectively.

In the naive large $N$ limit, $\omega$ is integer and $\omega$ should be restricted to satisfy $\omega-(d+l+$ $r[A]) \in 2 \mathbf{Z}$ for $L_{\omega l m}^{ \pm}$. This restriction comes from the fact that the spectrum are constructed by acting $P^{\mu}$ on the primary states. It is important to note that $L_{\omega l m}^{+}$correspond to the creation operators of the free gravity theory in the asymptotic $A d S_{d+1}$ [31] in the naive large $N$ limit [5].

\footnotetext{
${ }^{8}$ We ignored the Weyl anomaly here because it is a constant and does not play important roles in this paper.
} 
For a generic strong coupling large $N$ gauge theories with conformal symmetry, the low energy primary field is energy momentum tensor only and the spectrum generated by the it are expected to be independent as assumed in [5]. Thus, in the naive large $N$ limit, the (low energy) states are spanned by

$$
\left|\mathcal{N}_{A n l m}\right\rangle \equiv \prod_{A, n \in \mathbf{Z}_{\geq 0}, l, m} \frac{\left(L_{A \omega l m}^{+}\right)^{\mathcal{N}_{A \omega l m}}}{\left(\mathcal{N}_{A n l m}\right) !}|0\rangle
$$

where $\mathcal{N}_{A n l m}$ is a non-negative integer, $\omega=d+l+r[A]-2+2 n$ and

$$
\hat{H}\left(\prod_{A, \omega, l, m}\left(L_{A \omega l m}^{+}\right)^{\mathcal{N}_{A n l m}}\right)|0\rangle=\left(\sum_{A, n, l, m} \mathcal{N}_{A n l m} \omega\right)\left(\prod_{A, n, l, m}\left(L_{A \omega l m}^{+}\right)^{\mathcal{N}_{A n l m}}\right)|0\rangle .
$$

These coincide the states of the Fock space in the weak coupling limit of the gravity on AdS space as shown in [5]. These states are independent by the assumption in the limit taken in [5], however, will not be independent if we consider high-energy states for a large, but, finite $N$ case.

Including the $1 / N$ corrections, the conformal dimension of the energy momentum tensor is not modified, however, the dimension of the primary fields of multi trace operators will be modified slightly. Because this modification is small, the (low energy) states will be still spanned by energy eigen states labeled by $\left\{\mathcal{N}_{\text {Anlm }}\right\}$ :

$$
\left|\mathcal{N}_{\text {Anlm }}\right\rangle
$$

where we used same notation for the states in the naive large $N$ limit and their deformations by the $1 / N$ corrections. These eigen states $\left|\mathcal{N}_{\text {Anlm }}\right\rangle$ are also be generated by the primary (multi trace) fields and their descendants. This means that in the naive large $N$ limit the eigen state $\left|\mathcal{N}_{\text {Anlm }}\right\rangle$ reduces to the r.h.s. of (2.55). Note that the state $\left|\mathcal{N}_{\text {Anlm }}\right\rangle$ can not be simply given by (2.55) using an analogue of $L_{A \omega l m}^{+}$and the energy of $\left|\mathcal{N}_{A n l m}\right\rangle$ is no longer need to be an integer.

We will also define an deformation of $L_{A \omega l m}$ of the naive large $N$ limit to the case including the $1 / N$ corrections by

$$
\bar{L}_{A n l m} \equiv \frac{1}{2 \pi} \int_{-\pi}^{\pi} d \beta e^{-i(l+2 n) \beta} \sum_{\omega}\left(e^{i \beta}\right)^{\omega-d-r[A]+2} L_{A \omega l m},
$$

where $n \in \mathbf{Z} / 2$ and $\left.L_{A l m}\left(e^{i \beta}|x|\right)\right|_{|x|=1}=\sum_{\omega}\left(e^{i \beta}\right)^{\omega-d-r[A]+2} L_{A \omega l m}$ is the mode of the "time" translated energy momentum tensor $\left.\exp \left(i \beta|x| \frac{\partial}{\partial|x|}\right) T_{\mu \nu}(x)\right|_{|x|=1}$. In the cylinder coordinate, not in the radial quantization of the flat space, this indeed correspond to (formal) Fourier coefficient of the time translated energy momentum tensor, although the field is not periodic in time including $1 / N$ corrections. ${ }^{9}$ Because this is the "Fourier transformation", we can see that $\bar{L}_{A n l m}=\sum_{\omega \sim(d+l+r[A]-2+2 n)} L_{A \omega l m}+\mathcal{O}\left(1 / N^{2}\right)$ where $\omega \sim(d+l+r[A]-2+2 n)$

\footnotetext{
${ }^{9}$ This definition of a deformation of $L_{A \omega l m}$ will satisfy non-trivial commutators with the $1 / N$ corrections and reduce to the $L_{A \omega l m}$ in the large $N$ limit, although there are other definitions satisfying these properties.
} 
means that $\omega-(d+l+r[A]-2+2 n)=\mathcal{O}\left(1 / N^{2}\right)$, i.e. $\omega=d+l+r[A]-2+2 n$ in the naive large $N$ limit. Note that $\bar{L}_{A n l m}$ is not an eigen operator of the Hamiltonian $D$ and the violation of that is $\mathcal{O}\left(1 / N^{2}\right)$. The creation (and annihilation) operators in the naive large $N$ limit corresponds to the $\bar{L}_{A n l m}$ with $l \geq 2$ and $n \in \mathbf{Z}_{\geq 0}$ (and $n+d+l+r[A]-2 \in \mathbf{Z}_{\leq 0}$ ), respectively.

Before considering the commutation relation, we first investigate the singular parts of the OPE of the energy momentum tensors. For the energy momentum tensor, two point function is given by

$$
\left\langle T^{\mu_{1} \nu_{1}}(x) T^{\mu_{2} \nu_{2}}(0)\right\rangle=C_{T}\left(\frac{I_{\alpha}^{\mu_{1}}(x) I_{\beta}^{\nu_{l}}(x)\left(\frac{1}{2}\left(\delta_{\alpha \mu_{2}} \delta_{\beta \nu_{2}}+\delta_{\alpha \nu_{2}} \delta_{\beta \mu_{2}}\right)+\frac{1}{d} \delta_{\alpha \beta} \delta_{\mu_{2} \nu_{2}}\right)}{x^{2 d}}\right),
$$

where $I_{\nu}^{\mu}(x)=\delta^{\mu}{ }_{\nu}-2 \frac{x^{\mu} x_{\nu}}{x^{2}}$ and $C_{T}$ is a constant. We use the usual normalization for the energy momentum tensor in which the conformal generators are given by the mode expansions of the energy momentum tensor, like the Virasoro algebra. This means $C_{T}=$ $\mathcal{O}\left(N^{2}\right)$ for the large $N$ gauge theories. The three point function is also fixed in [24] with the three coefficients $\mathcal{A}, B, C$ with which $C_{T}$ is written as

$$
C_{T}=\frac{I(0)}{2} \frac{(d-1)(d+2) \mathcal{A}-2 \mathcal{B}-4(d+1) \mathcal{C}}{d(d+2)} .
$$

Note that the singular parts of the OPE between the energy momentum tensors are almost fixed, if the two and the three point functions are given, at least in principle, by the general argument of the CFT $[22,25,26]$. This is because the primary fields which can appear in the singular part are only the identity operator, the energy momentum tensor and the double trace operators, which correspond to composites of the two energy momentum tensors, if the anomalous dimensions of them are negative. We will neglect the multi trace operators for a while and consider them later. Thus, the singular part of the OPE is also given with only three unknown coefficients $A, B, C$ as

$$
\begin{aligned}
T^{\mu_{1} \nu_{1}}(x) T^{\mu_{2} \nu_{2}}(0)= & C_{T}\left(\frac{I_{\alpha}^{\mu_{1}}(x) I_{\beta}^{\nu_{l}}(x)\left(\frac{1}{2}\left(\delta_{\alpha \mu_{2}} \delta_{\beta \nu_{2}}+\delta_{\alpha \nu_{2}} \delta_{\beta \mu_{2}}\right)+\frac{1}{d} \delta_{\alpha \beta} \delta_{\mu_{2} \nu_{2}}\right)}{x^{2 d}}\right) \\
& +s_{\mu_{1} \nu_{1} \mu_{2} \nu_{2} \mu_{3} \nu_{3}}(x, \partial) T^{\mu_{3} \nu_{3}}(0)+\cdots,
\end{aligned}
$$

where $s_{\mu_{1} \nu_{1} \mu_{2} \nu_{2} \mu_{3} \nu_{3}}(x, \partial)$ consists of terms proportional to $\mathcal{A} / C_{T}, \mathcal{B} / C_{T}$ and $\mathcal{C} / C_{T}$. Because of the large $N$ factorization, we find $\mathcal{A}=\mathcal{O}\left(N^{2}\right), \mathcal{B}=\mathcal{O}\left(N^{2}\right)$ and $\mathcal{C}=\mathcal{O}\left(N^{2}\right) .{ }^{10}$

As for the scalar case, the commutation relations between the modes of the energy momentum tensor with the "time" derivatives $\frac{\partial^{p}}{\partial|x|^{p}} L_{A l m}(|x|)$ are fixed by the singular part of the OPE of the energy momentum tensor with the derivatives although we will not calculate them because of the technical difficulties. These commutation relations can be translated to the commutators betweens $\bar{L}_{A n l m}$ ordr by order, in principle. Thus, neglecting the contributions of the multi-trace operator, the commutators are given as

$$
\left[L_{i}, L_{j}\right]=N^{2} \omega_{i j} \mathbf{1}+f_{i j}^{k} L_{k}
$$

\footnotetext{
${ }^{10}$ We assumed that there are no low energy fields other than the energy momentum tensor.
} 
where $\omega_{i j}$ and $f_{i j}^{k}$ are $N$-independent constants and we denoted $\bar{L}_{A n l m}$ (or $\left.\left.\frac{\partial^{p}}{\partial|x|^{p}} L_{A l m}(|x|)\right|_{|x|=1}\right)$ as $L_{i}$, for short. These constants are, in principle, fixed by the OPE (2.61) which is fixed by the two and three point functions given in [24] which has only two parameters other than $N$. This algebra (2.62) forms an infinite dimensional Lie algebra including the identity operator 1 like the Virasoro algebra.

In the naive large $N$ limit, for the space of states (2.55), which are valid in the low energy approximation, any opeartor can be represented as a (formal) sum of the polynomials of the operators in $L^{+}$and $L^{-}$. Indeed, for a state $\left|\mathcal{N}_{A \omega l m}\right\rangle=\left(\prod_{A, n, l, m}\left(L_{A \omega l m}^{+}\right)^{\mathcal{N}_{A n l m}}\right)|0\rangle$ with the energy $E=\sum_{A, n, l, m} \mathcal{N}_{A n l m} \omega$, we can show $\left(\prod_{A, n, l, m}\left(L_{A \omega l m}^{+}\right)^{\mathcal{N}_{A n l m}^{\prime}}\right)^{\dagger}\left|\mathcal{N}_{A n l m}\right\rangle=0$ if $E^{\prime}=\sum_{A, n, l, m} \mathcal{N}_{A n l m}^{\prime} \omega>E$ because the vacuum is the lowest energy state. Furthermore, the states $\left|\mathcal{N}_{\text {Anlm }}\right\rangle$ which have a same energy are independent by the assumption. Thus, we can construct an operator $L$ which has the matrix element $\left\langle\mathcal{N}_{\text {Anlm }}|L| \mathcal{N}_{\text {Anlm }}\right\rangle$ from the polynomials of $L^{+}$and $L^{-}$order by order according to the energy of the ket. By replacing $L^{+}, L^{-}$to the corresponding operators in $\bar{L}_{A n l m}$ with non-negative integer $n$, above statements are clearly valid including the $1 / N$ corrections,

\section{Classical limit of the $C F T_{d}$}

In this section, we consider what is the classical limit of the large $N C F T_{d}$. For this, we will first introduce two more different normalizations of $L_{i}$. First, we define $L_{i}^{F}=\frac{1}{N} L_{i}$ which satisfy

$$
\left[L_{i}^{F}, L_{j}^{F}\right]=\omega_{i j} \mathbf{1}+\frac{1}{N} f_{i j}^{k} L_{k}^{F} .
$$

This normalization corresponds to the usual normalization of the primary field other than conserved currents, up to an $\mathcal{O}\left(N^{0}\right)$ factor, while $L_{i}$ include the conformal generators with an $\mathcal{O}\left(N^{0}\right)$ factor. This normalization is suitable especially for the free limit where we neglect the last term in (3.1), then they becomes the creation and annihilation operators (and $L^{\text {iso }}$ ) on the Fock space. The other normalization is defined by $L_{i}^{c l}=\frac{1}{N^{2}} L_{i}$ which satisfy

$$
N^{2}\left[L_{i}^{c l}, L_{j}^{c l}\right]=\omega_{i j} \mathbf{1}+f_{i j}^{k} L_{k}^{c l} .
$$

Note that the r.h.s. of this is $N$-independent. If we regard $N^{2}$ as $1 / \hbar$, it is expected, for $N \gg 1$, that

$$
-i\left\{L_{i}^{c l}, L_{j}^{c l}\right\}_{P}=\omega_{i j} \mathbf{1}+f_{i j}^{k} L_{k}^{c l}
$$

where $\left\{L_{i}^{c l}, L_{j}^{c l}\right\}_{P}$ is a Poisson bracket of the corresponding classical theory where $L_{i}^{c l}$ is identified as $\left\langle\Psi\left|L_{i}^{c l}\right| \Psi\right\rangle$ for a "classical" state $|\Psi\rangle$. We will see this below.

First, let us explain how to obtain the classical limit of the quantum mechanics, for example, for a particle. Note that a quantum mechanical system need not to have a classical limit if the theory is not obtained from a quantization of a classical system. Indeed, there exist purely quantum mechanical systems. On the other hand, let us consider a quantum mechanical system where we can choose operators $\hat{p}, \hat{x}$ with the commutation relations $-\frac{i}{\epsilon}\left[\hat{x}^{i}, \hat{p}_{j}\right]=\delta_{j}^{i}+\mathcal{O}(\epsilon)$, where $\epsilon$ is a small parameter, and the Hamiltonian $\hat{H}=\frac{1}{\epsilon} \hat{H}^{\prime}(\hat{p}, \hat{x})+$ 
$\mathcal{O}\left(\epsilon^{0}\right)$. Here, the time evolution of a operator $\hat{O}$ is assumed to be given by $\frac{d \hat{O}}{d t}=i[\hat{H}, \hat{O}] .{ }^{11}$ Then, regarding $\epsilon$ as $\hbar$, this quantum system may be derived by the quantization from the classical system with the Poisson bracket $\left\{x^{i}, p_{j}\right\}_{P}=\delta_{j}^{i}$ and the Hamiltonian $H^{c l}=\hat{H}^{\prime}$ which satisfies $\frac{d O}{d t}=-\left\{H^{c l}, O\right\}_{P}$. For this identification with the classical system, ${ }^{12}$ we need to consider a state with $x^{i}=\mathcal{O}\left(\epsilon^{0}\right)$ and $p_{j}=\mathcal{O}\left(\epsilon^{0}\right)$, which implies $H^{c l}=\mathcal{O}\left(\epsilon^{0}\right)$, and then this state will have large "quantum numbers" because $\epsilon$ is small.

This classical limit of the quantum system is an approximation, where states which have same $x^{i}, p_{j}$ up to $\mathcal{O}(\epsilon)$ differences are identified. For the operators, we need to consider the "classical operators" $\mathcal{O}\left(x^{i}, p_{j}\right)$ which are also defined up to $\mathcal{O}(\epsilon)$ differences. For the CFT case, $L_{i}^{c l}$ indeed correspond to $\hat{x}, \hat{p}$ above and give the classical limit with $\epsilon \sim 1 / N^{2}{ }^{13}$ Note that if we use $z^{a}(\hat{x}, \hat{p})$ as basis of operators instead of $\hat{x}, \hat{p}, \frac{i}{\epsilon}\left[\hat{x}^{i}, \hat{p}_{j}\right]=-\delta_{j}^{i}+\mathcal{O}(\epsilon)$ will be replaced by $\frac{i}{\epsilon}\left[z^{a}, z^{b}\right]=-f^{a b}(z)+\mathcal{O}(\epsilon)$ and these should satisfy the Jacobi identities. The commutation relations (3.2) are in this generalized form, locally.

Let us consider the contributions of the multi trace operators. The primary multi trace operators appear in the OPE between the energy momentum tensors, however, with $|x-y|^{m+\delta}$ where $m$ is non-negative integer and $\delta=\mathcal{O}\left(1 / N^{2}\right)$ is the anomalous dimension of the multi trace operator. Thus, the contributions to the commutator is suppressed by $\delta \sim 1 / N^{2}$. Indeed, if $\delta=0$ then this term is regular which can not contribute to the commutator. Next we will consider how much such the term with a multi trace operator in the OPE is suppressed in the large $N$ limit. First, we will consider scalar operators in the naive large $N$ limit for simplicity because essentially same considerations can be applied to the energy momentum case. The OPE is exactly given by

$$
\mathcal{O}_{\Delta_{a}}(x) \mathcal{O}_{\Delta_{b}}(y)=\frac{1}{(x-y)^{2 \Delta}} \delta_{a b}+:\left.\left(e^{z^{\mu} \frac{\partial}{\partial y^{\mu}}} \mathcal{O}_{\Delta_{a}}(y)\right)\right|_{z=x-y} \mathcal{O}_{\Delta_{b}}(y):
$$

where $: \cdots:$ is the free theory normal ordering, because it is a generalized free theory. Note that the $e^{z^{\mu} \frac{\partial}{\partial y^{\mu}}}$ generates just the descendants of $\mathcal{O}_{\Delta_{a}}$. The classical state for this is the coherent state $|\alpha\rangle$ for which the expectation value is $\left\langle\alpha\left|\mathcal{O}_{\Delta_{a}}(x)\right| \alpha\right\rangle=\alpha_{a}(x)$. Then, for the naive large $N$ limit where $\alpha_{a}(x)=\epsilon \mathcal{O}(N) \gg 1$, with $\epsilon \ll 1$,

$$
\begin{aligned}
\left\langle\alpha\left|\mathcal{O}_{\Delta_{a}}(x) \mathcal{O}_{\Delta_{b}}(y)\right| \alpha\right\rangle & =\frac{1}{(x-y)^{2 \Delta}} \delta_{a b}+\left\langle\alpha\left|:\left(e^{z^{\mu} \frac{\partial}{\partial y^{\mu}}} \mathcal{O}_{\Delta_{a}}(y)\right)\right|_{z=x-y} \mathcal{O}_{\Delta_{a}}(y): \mid \alpha\right\rangle \\
& \left.\sim\left(e^{z^{\mu} \frac{\partial}{\partial y^{\mu}}} \alpha_{a}(y)\right)\right|_{z=x-y} \alpha_{b}(y)=\alpha_{a}(x) \alpha_{b}(y)
\end{aligned}
$$

which is $\mathcal{O}\left(N^{2}\right)$ and the correct results in the classical limit. This implies that double trace operator terms appear in the $\mathrm{OPE}, \mathcal{O}_{\Delta_{a}}(x) \mathcal{O}_{\Delta_{b}}(y)$, including $1 / N$ corrections

\footnotetext{
${ }^{11}$ If we regard $\epsilon$ as $\hbar$, the usual definition of the Hamiltonian $\hat{H}^{\prime}(=\epsilon \hat{H})$ satisfies $\epsilon \frac{d \hat{O}}{d t}=i\left[\hat{H}^{\prime}, \hat{O}\right]$. Thus, our definition of the Hamitonian has a different normalization from the usual one. This is because we would like to consider the CFT where there is no notion of $\hbar$ generically.

${ }^{12}$ Note that if we start from the quantum mechanics, instead from the classical mechanics, the classical limit and the parameter $\hbar$ emerge if the theory satisfies some requirements. In particular we need a small parameter, which for our case $1 / N^{2} \sim 1 / C_{T}$.

${ }^{13}$ More precisely, $\left\{\frac{1}{N^{2}} L_{i}^{+c l}\right\}$ or $\left\{\left.\frac{\partial^{p}}{\partial r^{p}} T_{\mu \nu}(x)\right|_{r=1}\right\}$ generates any operator in the low energy states, thus they correspond to $x^{i}, p_{j}$.
} 
are suppressed by $1 / N$, at least, except $\left[\mathcal{O}_{\Delta_{a}} \mathcal{O}_{\Delta_{b}}\right](x)$. For the multi trace operator $\left[\prod_{m=1}^{p} \mathcal{O}_{\Delta_{a_{m}}}\right](x)$ in the OPE, this will gives $\mathcal{O}\left(N^{p}\right)$ contributions to $\left\langle\alpha\left|\mathcal{O}_{\Delta_{a}}(x) \mathcal{O}_{\Delta_{b}}(y)\right| \alpha\right\rangle$ where $|\alpha\rangle$ is a classical state. Such term is suppressed by $1 / N^{p-1}$, at least, for the consistency of the classical picture. ${ }^{14}$ Thus, including the suppression by the anomalous dimension $\delta$, in the classical limit, the multi trace operator contributions to the commutator is only the double trace operator $\left[\mathcal{O}_{\Delta_{a}} \mathcal{O}_{\Delta_{b}}\right](x)$ with an unknown parameter $\delta$. Therefore, the commtator including the multi trace operator contribution is

$$
N^{2}\left[L_{i}^{c l}, L_{j}^{c l}\right]=\omega_{i j} \mathbf{1}+f_{i j}^{k} L_{k}^{c l}+L_{k}^{c l} L_{l}^{c l} h_{i j}^{k l}+\mathcal{O}(1 / N),
$$

where $h_{i j}^{k l}$ is $\mathcal{O}\left(N^{0}\right)$ constant and the Poisson bracket is

$$
-i\left\{L_{i}^{c l}, L_{j}^{c l}\right\}_{P}=\omega_{i j} \mathbf{1}+f_{i j}^{k} L_{k}^{c l}+L_{k}^{c l} L_{l}^{c l} h_{i j}^{k l} .
$$

The commutator and the Poisson bracket should satisfy the Jacobi identities. For (3.7), this implies

$$
\sum f_{i j}^{l} \omega_{k l}=0, \sum\left(f_{i j}^{p} f_{k p}^{l}+2 h_{i j}^{l p} \omega_{k p}\right)=0
$$

where $\sum$ is taken over the cyclic permutations of $i, j, k$. Because only the unknown parameter are the anomalous dimensions of the primary double trace operators constructed from the energy momentum tensors, the last equation in (3.8) is expected to determine $h_{i j}^{k l}$. Therefore, the classical limit of the CFT is uniquely determined by the Poisson bracket (3.7) and the classical Hamiltonian $h_{c l}=\frac{1}{N^{2}} \hat{H}$ where $\hat{H}=D$.

We have seen that the classical limit of the $C F T_{d}$ may exist and is given by (3.7). The corresponding classical states for our case ${ }^{15}$ can be taken as the generalized coherent state which is a deformation of the Harmonic oscillator coherent state. The coherent state for the Harmonic oscillator is written as $e^{-|\alpha|^{2} / 2} \sum_{n=0}^{\infty} \frac{\alpha^{n}}{n !}|n\rangle$ where $|n\rangle$ is the normalized level $n$ state. The deformed coherent state for the perturbed Harmonic oscillator is obtained from the coherent state by replacing $|n\rangle$ to the eigen state of the perturbed Hamiltonian which is deformed from $|n\rangle$. This was used in [36, 37] although the commutator instead of the Hamiltonian is deformed for our case. Thus the deformed coherent state labeled by $\left\{\alpha_{A n l m}\right\}$ is

$$
\left|\alpha_{A n l m}\right\rangle=\prod_{A, n, l, m}\left[e^{-\left|\alpha_{A n l m}\right|^{2} / 2} \sum_{\mathcal{N}_{\text {Anlm }}=0}^{\infty} \frac{\left(\alpha_{\text {Anlm }}\right)^{\mathcal{N}_{\text {Anlm }}}}{\mathcal{N}_{\text {Anlm }} !}\right]\left|\mathcal{N}_{A n l m}\right\rangle,
$$

where $\alpha_{\text {Anlm }}$ is $\mathcal{O}(N)$ constant. This deformed coherent state may be regarded as the classical state. at least, for $\alpha_{A n l m} / N \ll 1$.

Until now, we have only used the properties of generic large $N$ gauge theories with the conformal symmetry to derive the classical limit of the CFT. It is expected that this classical system is identified as the classical (Einstein) gravity on asymptotic $A d S_{d+1}$ space because the classical system certainly reduces to the linearized gravity in the large $N$ limit taken in [5]. We will show this identification is indeed correct in the next section.

\footnotetext{
${ }^{14}$ We expect that this suppression is explained by the large $N$ factorization.

${ }^{15}$ If we can neglect the multi trace operators, we can use the generalized coherent states based on Lie groups [32-34]. It might be possible to incorporate the contributions of multi trace operators with minor modifications to this method, we will explain the generalized coherent state based on (3.2) in the appendix.
} 
Finally, we note that the this classical description is, of course, an approximation. In particular, the Hilbert space we consider is the low energy approximation and the classical approximation will be violated if the energy of the states are sufficiently large. This bound of the energy for the classical approximation is (less than) $\mathcal{O}\left(N^{2}\right)$ which is the degrees of freedom of the gauge theory.

\section{Classical gravity on asymptotic $A d S_{d+1}$ and the $C F T_{d}$}

In this section, we will consider the classical gravity on asymptotic $A d S_{d+1}$ in the Hamiltonian formalism, in particular using the Brown-York tensor. We will see that the classical dynamics of generic large $N$ gauge theories with the conformal symmetry is equivalent to the Einstein gravity on asymptotic $A d S_{d+1}$. (We will explain the difference between the large $N$ expansion from the free theory and the classical limit in the appendix A.)

The Einstein-Hilbert action of the gravitational theory, with appropriate boundary terms, is

$$
S_{\text {grav }}=\frac{1}{2\left(l_{p}\right)^{d-1}} \int_{A d S_{d+1}} d^{d+1} x \sqrt{-\operatorname{det} g}(R-2 \Lambda)+S_{G H}+S_{c t},
$$

where $\left(l_{p}\right)^{d-1}=8 \pi G_{N}, \Lambda=-\frac{d(d-1)}{2 l_{\mathrm{AdS}}^{2}}$ and we set the AdS scale $l_{\mathrm{AdS}}=1 \mathrm{in}$ this section. The Gibbons-Hawking term $S_{G H}$ is needed to allow the Dirichlet boundary condition as a consistent boundary condition and $S_{c t}$ is needed for making the action finite [38, 39], although this term does not play any role in the equations of motion of the classical dynamics which we concentrate on this paper. The metric of the vacuum solution is the $A d S_{d+1}$ metric:

$$
d s^{2}=g_{\mu \nu}^{\mathrm{AdS}} d x^{\mu} d x^{\nu}=-\left(1+r^{2}\right) d t^{2}+\frac{1}{1+r^{2}} d r^{2}+r^{2} d \Omega_{d-1}^{2}
$$

where $0 \leq r<\infty,-\infty<t<\infty$ and $d \Omega_{d-1}^{2}$ is the metric for the $d$-1-dimensional round unit sphere $S^{d-1}$. Let us parametrize the metric as $g_{\mu \nu}=g_{\mu \nu}^{\mathrm{AdS}}+h_{\mu \nu}$ and consider $h_{\mu \nu}$ as the varying fields.

First, we consider the free limit of the gravity, i.e. the linearized gravity. The e.o.m. of this limit was explicitly solved in [31] using the gauge invariant combinations. Because this is free theory, we can easily see that in the (classical) Hamiltonian formalism, the results can be expressed as

$$
\left\{a_{A n l m}, a_{A^{\prime} n^{\prime} l^{\prime} m^{\prime}}^{\dagger}\right\}_{P}=\delta_{A, A^{\prime}} \delta_{n, n^{\prime}} \delta_{l, l^{\prime}} \delta_{m, m^{\prime}}
$$

and

$$
h_{c l}=\sum_{A, n, l, m} \omega a_{A n l m}^{\dagger} a_{A n l m}
$$

where $(A, n, l, m)$ are the labels for the $L_{A \omega l m}^{+}$where $\omega=d+l+r[A]-2+2 n[5]$. Here, we require that the only the normalizable modes of $h_{\mu \nu}$ are dynamical and the non-normalizable modes of $h_{\mu \nu}$ are set to be zero, which corresponds to fixing the boundary conditions. This classical system is equivalent to the system with the Poisson bracket (3.3) and the Hamiltonian as the dilatation if we neglect the terms proportional to $f_{i j}^{k}$, which are $1 / N$ corrections to the free limit. 
If we consider the full Einstein gravity, we need to include the non-linear interactions of the modes of $h_{\mu \nu}$. Then, in the ADM formalism with an appropriate gauge fixing, we will have a Poisson bracket and a Hamiltonian which are modified by some $G_{N}$ corrections from the ones for free cases, i.e. (4.3) and (4.4). It is possible to determine the corrections for the Hamiltonian explicitly, in principle, by the perturbation in $G_{N}$, but difficult practically. However, we note that the independent variables (which need not to be canonical) may be labeled by same indices $(A, n, l, m)$ for the free case and we will denote them as $a_{A n l m}$ even for the interacting case because the interactions do not change the independent variables of the classical theory. ${ }^{16}$

It is important to note that this system have the symmetry corresponding to the isometries of $A d S_{d+1}$ with the metric $g_{\mu \nu}^{\text {AdS }}$, whose generators can be constructed by the Noether method or the asymptotic symmetry group, see, for example, [40]. Of course this symmetry is isomorphic to the conformal group of $C F T_{d}$ and includes the Hamiltonian as the dilatation.

Moreover, we can construct the analogue of the energy momentum tensor of $C F T_{d}$ in this system, $T_{\mu \nu}^{b n d y}(x)$, which is called the boundary stress tensor, or the Brown-York tensor $[38,39,41]$. The boundary stress tensor is defined as

$$
T_{b n d y}^{\mu \nu}(x)=\frac{2}{\sqrt{-\operatorname{det} g_{\mu \nu}^{(0)}}} \frac{\delta S_{\text {grav }}}{\delta g_{\mu \nu}^{(0)}(x)},
$$

which is defined on the boundary of the asymptotically $A d S_{d+1}$, where $g_{\mu \nu}^{(0)}(x)$ is the boundary metric and $\mu, \nu$ runs for the tangent directions of the boundary. ${ }^{17}$ It was shown that this boundary stress tensor is conserved, i.e. $\nabla_{\mu} T_{b n d y}^{\mu \nu}(x)=0$ where $\nabla_{\mu}$ is the covariant derivative on the boundary because of the diffeomorphism invariance of the action [40,41]. It was also shown that the trace of the boundary stress tensor vanishes for odd $d$ and a constant for even $d[38,39,42]$. This constant corresponds to the conformal anomaly if we assume the AdS/CFT. Here, it is important that this constant is fixed if we fix the boundary metric, thus we can neglect this constant in the Poisson bracket. In summary, the (traceless part of the) boundary stress tensor is symmetric, traceless and conserved. Furthermore, this tensor will transform as a primary field by the conformal symmetry transformation. This is because the symmetry is associate with the diffeomorphism which induces the conformal transformation on the boundary metric $g_{\mu \nu}^{(0)}$. With the Killing vector field for these diffeomorphisms, the conformal symmetry generators are given by the boundary stress tensor as usual. Thus, the boundary stress tensor $T_{b n d y}^{\mu \nu}(x)$ is regarded as an energy-momentum tensor of a $C F T_{d}$ where the commutators are defined by the Poissson bracket .

In the Hamiltonian or ADM formalism with a gauge fixing, which corresponds to a coordinate choice, the action $S_{\text {grav }}$ and the boundary stress tensor $T_{b n d y}^{\mu \nu}$ are functions of the variables $a_{A n l m}$ and $a_{A n l m}^{\dagger}$ where we fix the boundary metric $g_{\mu \nu}^{(0)}$ as the cylinder. We

\footnotetext{
${ }^{16}$ Which values the indices $(A, n, l, m)$ take are also same for the free case.

${ }^{17}$ To define the boundary stress tensor more precisely, we first introduce the IR cut-off for the radial direction $r$ and then remove the cut-off. The boundary metric is defined by removing the warped factor. Details of the construction, see [38-40].
} 
can expand $T_{b n d y}^{\mu \nu}$ by the symmetric tensor harmonics and obtain the corresponding modes $L_{A l m}^{b n d y}(|x|)$ and $\bar{L}_{A n l m}^{b n d y}$ as in the previous section. ${ }^{18}$ Thus, the modes $\bar{L}_{A n l m}^{b n d y}$ are functions of $a_{A \omega l m}$ and $a_{A \omega l m}^{\dagger}$. Conversely, the variables $\left\{a_{A n l m}, a_{A n l m}^{\dagger}\right\}$ can be regarded as functions of $\bar{L}_{A n l m}^{\text {bndy }}$ where $l \geq 2$ and $n \in \mathbf{Z}_{\geq 0}$ or $n+d+l+r[A]-2 \in \mathbf{Z}_{\leq 0}$, i.e. the creation and annihilation operators, (at least if the theory is close to the free theory) because the number of the independent variables are same. We can regard the map between these two as a field redefinition although in order to obtain such a map explicitly we need to solve the equations of motion. Note that the modes defined at the boundary can be equivalent to the whole bulk modes because the diffeomorphism gives the constraints and the system in the AdS space is like the system in a box [40, 43].

Therefore, the Poisson bracket algebra, which satisfies the Jacobi identities, of the boundary stress tensor is same as the algebra of the energy momentum tensor with the three parameters. However, if we require the unitarity and the causality (and the sparseness of the spectrum which we already assumed), ${ }^{19}$ it have been shown $[45,46]$ that there remains only one parameter $C_{T}$ in (2.61) which is $\mathcal{O}\left(N^{2}\right)$ for the gauge theory. For the $T_{b n d y}^{\mu \nu}$, we can see $C_{T} \sim 1 / G_{N}$. This is because $T_{b n d y}^{\mu \nu} \sim 1 / G_{N}$ by definition and $\{h, \dot{h}\}_{P} \sim G_{N}$ where $h$ is the some component of $h_{\mu \nu}$, schematically. We can fix the coefficient of this relation by computing above precisely for the free theory limit and the result should agree with the result assuming the AdS/CFT correspondence as $C_{T}=\frac{d+1}{d-1} \frac{\Gamma(d+1)}{\pi^{d / 2} \Gamma(d-1)}\left(\frac{l_{\mathrm{AdS}}}{l_{p}}\right)^{d-1}$ [44]. The Hamiltonian of this gravitational system can be identified as the dilatation in the conformal symmetry. Thus, we conclude that the classical limit of the generic large $N$ gauge theory with conformal symmetry is the classical Einstein gravity on asymptotic $A d S_{d+1}$ because the Hamiltonian and the Poisson bracket are same.

\section{Acknowledgments}

S.T. would like to thank Kanato Goto, Shigeki Sugimoto and Sotaro Sugishita for useful discussions. S.T. would like to thank Yu Nakayama for important comments and discussions. This work was supported by JSPS KAKENHI Grant Number 17 K05414.

\section{A Classical limit of $A d S / C F T$ and coherent state}

In this section, we will explain how to take the classical gravity limit in terms of the large $N$ CFT assuming the AdS/CFT correspondence although this appendix is not used in the main parts of this paper. We will also explain the (generalized) coherent states, which are not used in the main parts of this paper.

\footnotetext{
${ }^{18}$ This expansion might not be guaranteed to be valid in general. However, at least, in the perturbation in $G_{N}$, we expect that such expansion is possible.

${ }^{19}$ If we do not require these, then the Gauss-Bonnet gravity, and more generally the Lovelock gravity may correspond to the theory with more parameters. Note that the Lovelock gravity are the most general metric theory of gravity yielding second order equations of motion, which is need to keep the number of the dynamical variables. For the Gauss-Bonnet gravity, the parameters are identified in [44].
} 
Let us consider the following metric around the AdS space:

$$
d s^{2}=g_{\mu \nu} d x^{\mu} d x^{\nu}=\left(l_{\mathrm{AdS}}\right)^{2}\left(g_{\mu \nu}^{\mathrm{AdS}} d x^{\mu} d x^{\nu}+h_{\mu \nu} d x^{\mu} d x^{\nu}\right),
$$

where $l_{\mathrm{AdS}}$ is the AdS (length) scale and $h_{\mu \nu}$ is the fluctuation around the AdS space.

The action of the gravitational theory in the derivative expansion is, schematically,

$$
S_{\text {grav }}=\frac{1}{2\left(l_{p}\right)^{d-1}} \int d^{d+1} x \sqrt{-\operatorname{det} g}\left(-\frac{d(d+1)}{2 l_{\mathrm{AdS}}^{2}}+R+\alpha_{1} D^{2} R+\alpha_{2} l_{\mathrm{AdS}}^{2} R^{2}+\cdots\right),
$$

where $\left(l_{p}\right)^{d-1}=8 \pi G_{N}, \alpha_{i}$ are dimensionless constants and $x^{\nu}$ and $h_{\mu \nu}$ are also dimensionless. We assume that the AdS space is the solution of the e.o.m. of this action, as for the Gauss-Bonnet gravity or $g_{\mu \nu}^{\mathrm{AdS}}$ is modified from the AdS metric to be the solution with the higher derivative terms.

Let us define the dimensionless parameter $N$ as $N^{2}=\left(\frac{l_{\mathrm{AdS}}}{l_{p}}\right)^{d-1}$, then, the action for the fluctuation is, schematically, given by

$$
\begin{aligned}
S= & N^{2} \int d^{d+1} x \sqrt{-\operatorname{det}\left(g^{\mathrm{AdS}}\right)} \\
& \times\left(-f_{0}(h) h^{2}+f_{1}(h)(D h)^{2}+\alpha_{1} f_{2}(h)(D D h)^{2}+\alpha_{2} f_{3}(h)(D h)^{4}+\cdots\right),
\end{aligned}
$$

where we abbreviated the various contractions of the indices and $f_{i}(h)$ is a function such that $f(h=0)$ is finite.

There are several choices for large $N$ limits. One is the perturbation around the AdS geometry or the liner approximation. For this, we will normalize $h_{\mu \nu}$ such that the kinetic term will be the canonical one. Thus, we need to take $\tilde{h}_{\mu \nu} \sim N h_{\mu \nu}$ small, but finite. If the higher derivative terms vanish, i.e. $\alpha_{i} \rightarrow 0$, in the large $N$ limit, we have the free theory of $\tilde{h}$ in the leading order in this large $N$ limit, with the spectrum given in [31] corresponding to the energy momentum tensor of the CFT [5]. Thus, $\tilde{h}$ is directly related to the creation/annihilation operators of the free theory. The sub-leading terms are interactions which include the terms in $R, D^{2} R$ and so on. In general, this $1 / N$ expansion with $\tilde{h}$ include the classical and the quantum gravity effects.

Another choice of the large $N$ limit is the classical limit where $h_{\mu \nu}$ is finite and $N^{2}$ is regarded as $1 / \hbar$. This means that the v.e.v. of the creation/annihilation operators, i.e. $\tilde{h}$, should have $\mathcal{O}(N)$ values. Note that this large $N$ limit contains the whole interactions of the Einstein gravity as a leading term, which are non-leading term in the previous $1 / N$ expansion with $\tilde{h}$. In this paper, we will consider this large $N$ limit in the CFT.

\section{A.1 Coherent states for the linearized gravity}

We can consider classical states which is very close to the vacuum, i.e. $h_{\mu \nu}=\mathcal{O}\left(N^{0}\right)$, but $h_{\mu \nu} \sim \epsilon \ll 1$. This is the linear approximation of the Einstein gravity (at least if $\alpha_{i} \rightarrow 0$ in the large $N$ limit). Here, we describe the coherent states for this approximation, which have been considered in [47-50].

Let us remember the coherent states for the free field (harmonic osscilator). The coherent state for it is defined as $|\alpha\rangle=e^{\alpha a^{\dagger}-\bar{\alpha} a}|0\rangle$, where $\left[a, a^{\dagger}\right]=1$ and $a|0\rangle=0$, which 
satisfies $a|\alpha\rangle=\alpha|\alpha\rangle$ and $\int_{\mathbf{C}} d^{2} \alpha|\alpha\rangle\langle\alpha|=\pi$. The overlap between the coherent state and the normalized energy eigen state $|n\rangle=\frac{1}{n !}\left(a^{\dagger}\right)^{n}|0\rangle$ is given by

$$
\langle n \mid \alpha\rangle=e^{-\frac{1}{2}|\alpha|^{2}} \frac{\alpha^{n}}{\sqrt{n !}}=e^{-\frac{1}{2}|\alpha|^{2}+f(n)+\mathcal{O}(\ln n)},
$$

where $f(n)=-\frac{1}{2} n(\ln n-2 \ln \alpha-1)$. Thus, particle numbers where dominant contributions comes from for the coherent state is $n \sim \alpha^{2}$ because $\frac{\partial f(n)}{\partial n}=0$ at $n=\alpha^{2}$.

Therefore, the classical state which is very close to the vacuum in the CFT is

$$
\left|\beta^{i}\right\rangle=e^{N \epsilon \sum_{i}\left(\beta^{i} a_{i}^{\dagger}-\bar{\beta}^{i} a_{i}\right)}|0\rangle,
$$

where $\beta^{i}=\mathcal{O}\left(N^{0}\right)$ are complex constants, $\epsilon$ is a small parameter and $a_{i}^{\dagger}$ is the creation operator (2.10). This state is expected to be the coherent state $a_{i}\left|\beta^{i}\right\rangle \sim N \epsilon \beta^{i}\left|\beta^{i}\right\rangle$, where we assumed $\left[a_{i}, a_{j}^{\dagger}\right]=\delta_{i j}+\mathcal{O}\left(N^{-1}\right)$ and the $\mathcal{O}\left(N^{-1}\right)$ terms are neglected in the small $\epsilon$ limit. The time evolution (in the Schrödinger picture) is given by $i \frac{\partial}{\partial t}\left|\beta^{i}\right\rangle=\hat{H}\left|\beta^{i}\right\rangle=D\left|\beta^{i}\right\rangle$ where $D$ is the dilatation operator and $\left[D, a_{i}\right]=\omega_{i} a_{i}$. Thus, the solution is $\left|\beta^{i}(t)\right\rangle=$ $e^{N \epsilon \sum_{i}\left(\beta^{i}(t) a_{i}^{\dagger}-\bar{\beta}^{i}(t) a_{i}\right)}|0\rangle$, where $\frac{\partial \beta_{i}(t)}{\partial t}=\omega_{i} \beta_{i}(t) \cdot{ }^{20}$

\section{A.2 Coherent states for (3.2)}

For the Lie algebra (3.2), we can use the generalized coherent states based on Lie groups [3234]. In this section, we assume (3.2) instead of (3.6). We basically follows [35] to consider the generalized coherent states and their properties. ${ }^{21}$ First, we define the coherent group $G$ which consist of the following unitary operators:

$$
\hat{U}=e^{i\left(c^{i} L_{i}+c N^{2} \mathbf{1}\right)}=e^{i N^{2}\left(c^{i} L_{i}^{c l}+c \mathbf{1}\right)},
$$

where $L_{i}$ are taken to be Hermite and $c^{i}, c$ are $N$-independent real constants. We also define the isotropy subgroup $H$ of the coherent group whose element $\hat{V}$ satisfies $\hat{V}|0\rangle=|0\rangle$ up to a phase factor, i.e. $\hat{V}=e^{i\left(c^{i} L_{i}^{\text {iso }}+c N^{2} 1\right)}$.

Then, the generalized coherent states are defined by $\hat{U}|0\rangle$ with parameters $c^{i}, c$ in (A.6). ${ }^{22}$ Using the Baker-Campbell-Hausdorff formula, we can rewrite it as

$$
\hat{U}|0\rangle=C e^{\alpha^{i} L_{i}^{+}}|0\rangle,
$$

where $\alpha^{i}$ are some complex functions of $c^{i}, c$ which are $N$-independent and $C=$ $\left.e^{i N^{2} \theta}\left|e^{\alpha^{i} L_{i}^{+}}\right| 0\right\rangle\left.\right|^{-\frac{1}{2}}$ where $\theta$ is a $N$-independent real complex function of $c^{i}, c$. Thus, generalized coherent states are parametrized by $\alpha^{i}$ and the states with same $\alpha^{i}$ should be identified. ${ }^{23}$

\footnotetext{
${ }^{20}$ We can replace $|0\rangle$ to any state in this. Indeed, $a_{i}$ is assumed to be diagonalized by the Hamiltonian, i.e. $D$, thus $a_{i}$ is the solution of the e.o.m.

${ }^{21}$ There could be some differences between the large $N$ limit taken in [35] and this paper. The large $N$ limit in [35] seems to correspond to the free limit because the factorization of the correlation functions were discussed. Here, (3.2) include the $1 / N$ corrections which violate the factorization properties.

${ }^{22}$ Because the Lie algebra is infinite dimensional, we need to require some properties for $c_{i}$ such that the coherent state is well-defined, in particular, the state should have a finite energy. A simple requirement for this is that only a finite number of $c_{i}$ do not vanish. This will be too strong condition and it is desirable to find an appropriate condition although we just assume the state is well-defined in this paper.

${ }^{23}$ The coherent states are parametrized by the coadjoint orbit as we will see later.
} 
We can also see that the classical operators defined in [35] are the operators constructed from $L_{i}^{c l}$. In order to see this, let us consider two coherent states $\hat{U}|0\rangle=C e^{\alpha^{i} L_{i}^{+}}|0\rangle$ and $\hat{U}^{\prime}|0\rangle=C^{\prime} e^{\left(\alpha^{\prime i} L_{i}^{+}\right)}|0\rangle$ where $\hat{U}, \hat{U}^{\prime} \in G$. Then, $\left.\hat{U}^{\dagger} \hat{U}^{\prime}|0\rangle=e^{i N^{2} \theta_{c}} e^{\alpha_{c}^{i} L_{i}^{+}}|0\rangle\left|e^{\alpha_{c}^{i} L_{i}^{+}}\right| 0\right\rangle\left.\right|^{-\frac{1}{2}}$ is also a coherent state and the overwrap of the two coherent states is given by $\left\langle 0\left|\hat{U}^{\dagger} \hat{U}^{\prime}\right| 0\right\rangle=$ $\left.\left.e^{i N^{2} \theta_{c}}\left\langle 0\left|e^{\alpha_{c}^{i} L_{i}^{+}}\right| 0\right\rangle\left|e^{\alpha_{c}^{i} L_{i}^{+}}\right| 0\right\rangle\left.\right|^{-\frac{1}{2}}=e^{i N^{2} \theta_{c}}\left|e^{\alpha_{c}^{i} L_{i}^{+}}\right| 0\right\rangle\left.\right|^{-\frac{1}{2}}$. By the Baker-Campbell-Hausdorff formula, we expect the following rewriting: $e^{\bar{\alpha}_{c}^{i} L_{i}^{-}} e^{\alpha_{c}^{i} L_{i}^{+}}=e^{\beta^{i} L_{i}^{+}} e^{\gamma^{i} L_{i}^{\text {iso }}+\phi N^{2}} e^{\bar{\beta}^{i} L_{i}^{-}}$where $\beta^{i}, \gamma^{i}, \phi$ are some $N$-independent constants determined by $\alpha^{i}$. Using this expression, we have $\mathcal{R} e\left(\ln \left\langle 0\left|\hat{U}^{\dagger} \hat{U}^{\prime}\right| 0\right\rangle\right)=-N^{2} \phi / 2$ where $\mathcal{R} e$ means the real part. Then, if $\alpha_{c}^{i}$ is non zero for some $i, \phi>0$ because $\left|\left\langle 0\left|\hat{U}^{\dagger} \hat{U}^{\prime}\right| 0\right\rangle\right|<1$. Similarly, we can also show that $\mathcal{R} e\left(\ln \left\langle 0\left|\hat{A}_{c l} \hat{U}^{\dagger} \hat{U}^{\prime}\right| 0\right\rangle\right)=-N^{2} \phi / 2+\mathcal{O}\left(N^{0}\right)$ where $\hat{A}_{c l}$ is constructed from $L_{i}^{c l}$ without $N$ dependent coefficients. This means that $\mathcal{R} e\left(\ln \left\langle 0\left|\hat{U}^{\dagger} \hat{A}_{c l} \hat{U}^{\prime}\right| 0\right\rangle\right)=-N^{2} \phi / 2+\mathcal{O}\left(N^{0}\right)$ because $\hat{U} \hat{A}_{c l} \hat{U}^{\dagger}=\hat{\tilde{A}}_{c l}$ where $\hat{\tilde{A}}_{c l}$ also is an operator constructed from $L_{i}^{c l}$ without $N$-dependent coefficients.

The classical operators defined in [35] are the operators, say, $\hat{A}$ such that $\left\langle 0\left|U^{\dagger} \hat{A} U\right| 0\right\rangle /\left\langle 0\left|\hat{U}^{\dagger} \hat{U}^{\prime}\right| 0\right\rangle$ is finite in the $N \rightarrow \infty$ limit. Therefore, we find that the classical operators are indeed the operators constructed from $L_{i}^{c l}$.

We can also easily show that two coherent states, $\hat{U}|0\rangle=C e^{\left(\alpha^{i} L_{i}^{+}\right)}|0\rangle$ and $\hat{U}^{\prime}|0\rangle=$ $C^{\prime} e^{\left(\alpha^{\prime i} L_{i}^{+}\right)}|0\rangle$, are classically equivalent [35], which means $\left\langle 0\left|\hat{U}^{\dagger} \hat{A}_{c l} \hat{U}\right| 0\right\rangle=\left\langle 0\left|\hat{U}^{\prime \dagger} \hat{A}_{c l} \hat{U}^{\prime}\right| 0\right\rangle$ for any $\hat{A}_{c l}$, if $\alpha^{i}=\alpha^{i}$. Even if $\alpha^{i} \neq \alpha^{i}$, two states can be classically equivalent. Including such identification, the coherent states are parametrized by the coadjoint orbit. We will shortly explain this below. First, denoting $g$ as the Lie algebra of the coherent group $G$, we can define the dual space $g^{*}$ whose elements are linear functionals acting on $g$. Then, the expectation values of $L_{i}^{c l}$ for a coherent state, $\left\langle 0\left|\hat{U}^{\dagger} L_{i}^{c l} \hat{U}\right| 0\right\rangle$, can be regarded as an element $\zeta^{\hat{U}}$ in $g^{*}$ if we regard $L_{i}^{c l}$ as basis of $g$. In particular, we will denote $\zeta^{\mathbf{1}}$ as for the element corresponding to $|0\rangle$ for which the components are given by $\zeta_{i}^{1}=\left\langle 0\left|L_{i}^{c l}\right| 0\right\rangle$. The coadjoint orbit $\Gamma$ is the set of $\zeta^{\hat{U}}$ in $g^{*}$ generating by $\hat{U}$. Note that the expectation values of the operators constructed from $L_{i}^{c l}$ are fixed by the $\zeta^{\hat{U}}$ because of the factorization of the expectation values [35]. Thus, classical equivalence class of the coherent states are identified as the coadjoint orbit ${ }^{24}$ and then, the classical phase space is identified as the coadjoint orbit.

There are some requirements [35] such that the classical limit considered here is indeed behaves as the classical dynamics. The one is the irreducibility of the representation of $G$ and this is satisfied because we assumed (2.55) are all independent and our algebra reduced to the free harmonic oscillators, i.e. Heisenberg algebras, for the small $c^{i}, c$. It also required that if $\left\langle 0\left|U^{\dagger} \hat{A} U\right| 0\right\rangle=0$ for any $U, U^{\prime} \in G$, then $\hat{A}=0$. This is also satisfied. The requirement about the overwrap between the coherent states was already shown to be satisfied above. The last requirement is about the Hamiltonian. The classical Hamiltonian $h_{c l}$ is given by $h_{c l}=\frac{1}{N^{2}} \hat{H}$, where $\hat{H}=D$ is the Hamiltonian in our theory, and $h_{c l}$ is indeed the classical operator. Here, in the classical limit $h_{c l}$ is regarded as a function on the coadjoint orbit. Thus, all the requirements are satisfied and we conclude that the (classical)

\footnotetext{
${ }^{24}$ The coadjoint orbit is parametrized by the possible (expectation) values of $L_{i}^{c l}$, although $L_{i}^{c l}$ for $L^{\text {iso }}$ are not independent.
} 
equations of motion for a function $f$ on the coadjoint orbit, which are parametrized by $L_{i}^{c l}$, is

$$
\frac{d}{d t} f=\left\{h_{c l}, f\right\}_{P},
$$

with the Poisson bracket (3.3) for the classical limit of the CFT.

Open Access. This article is distributed under the terms of the Creative Commons Attribution License (CC-BY 4.0), which permits any use, distribution and reproduction in any medium, provided the original author(s) and source are credited.

\section{References}

[1] J.M. Maldacena, The large $N$ limit of superconformal field theories and supergravity, Int. J. Theor. Phys. 38 (1999) 1113 [hep-th/9711200] [INSPIRE].

[2] S.S. Gubser, I.R. Klebanov and A.M. Polyakov, Gauge theory correlators from noncritical string theory, Phys. Lett. B 428 (1998) 105 [hep-th/9802109] [INSPIRE].

[3] E. Witten, Anti-de Sitter space and holography, Adv. Theor. Math. Phys. 2 (1998) 253 [hep-th/9802150] [INSPIRE].

[4] T. Banks, M.R. Douglas, G.T. Horowitz and E.J. Martinec, AdS dynamics from conformal field theory, hep-th/9808016 [INSPIRE].

[5] S. Terashima, AdS/CFT correspondence in operator formalism, JHEP 02 (2018) 019 [arXiv: 1710.07298] [INSPIRE].

[6] V. Balasubramanian, P. Kraus and A.E. Lawrence, Bulk versus boundary dynamics in anti-de Sitter space-time, Phys. Rev. D 59 (1999) 046003 [hep-th/9805171] [INSPIRE].

[7] I. Heemskerk, J. Penedones, J. Polchinski and J. Sully, Holography from conformal field theory, JHEP 10 (2009) 079 [arXiv: 0907.0151] [INSPIRE].

[8] A.L. Fitzpatrick and J. Kaplan, AdS field theory from conformal field theory, JHEP 02 (2013) 054 [arXiv: 1208.0337] [INSPIRE].

[9] I. Bena, On the construction of local fields in the bulk of $A d S_{5}$ and other spaces, Phys. Rev. D 62 (2000) 066007 [hep-th/9905186] [INSPIRE].

[10] A. Hamilton, D.N. Kabat, G. Lifschytz and D.A. Lowe, Local bulk operators in AdS/CFT: a boundary view of horizons and locality, Phys. Rev. D 73 (2006) 086003 [hep-th/0506118] [INSPIRE].

[11] A. Hamilton, D.N. Kabat, G. Lifschytz and D.A. Lowe, Holographic representation of local bulk operators, Phys. Rev. D 74 (2006) 066009 [hep-th/0606141] [INSPIRE].

[12] S. El-Showk and K. Papadodimas, Emergent spacetime and holographic CFTs, JHEP 10 (2012) 106 [arXiv:1101.4163] [INSPIRE].

[13] D. Kabat, G. Lifschytz and D.A. Lowe, Constructing local bulk observables in interacting AdS/CFT, Phys. Rev. D 83 (2011) 106009 [arXiv:1102.2910] [InSPIRE].

[14] D. Kabat, G. Lifschytz, S. Roy and D. Sarkar, Holographic representation of bulk fields with spin in AdS/CFT, Phys. Rev. D 86 (2012) 026004 [arXiv:1204.0126] [INSPIRE].

[15] D. Kabat and G. Lifschytz, CFT representation of interacting bulk gauge fields in AdS, Phys. Rev. D 87 (2013) 086004 [arXiv: 1212.3788] [INSPIRE]. 
[16] A.L. Fitzpatrick, J. Kaplan and M.T. Walters, Universality of long-distance AdS physics from the CFT bootstrap, JHEP 08 (2014) 145 [arXiv: 1403.6829] [INSPIRE].

[17] D. Kabat and G. Lifschytz, Bulk equations of motion from CFT correlators, JHEP 09 (2015) 059 [arXiv:1505.03755] [INSPIRE].

[18] D. Kabat and G. Lifschytz, Locality, bulk equations of motion and the conformal bootstrap, JHEP 10 (2016) 091 [arXiv: 1603.06800] [INSPIRE].

[19] M. Duetsch and K.-H. Rehren, Generalized free fields and the AdS-CFT correspondence, Annales Henri Poincaré 4 (2003) 613 [math-ph/0209035] [INSPIRE].

[20] G. 't Hooft, On the quantum structure of a black hole, Nucl. Phys. B 256 (1985) 727 [INSPIRE].

[21] N. Iizuka and S. Terashima, Brick walls for black holes in AdS/CFT, Nucl. Phys. B 895 (2015) 1 [arXiv:1307.5933] [INSPIRE].

[22] D. Simmons-Duffin, The conformal bootstrap, in Proceedings, Theoretical Advanced Study Institute in Elementary Particle Physics: new frontiers in fields and strings (TASI 2015), Boulder, CO, U.S.A., 1-26 June 2015, World Scientific, Singapore (2017), pg. 1 [arXiv: 1602.07982] [INSPIRE].

[23] J. Avery, Hyperspherical harmonics; applications in quantum theory, Kluwer Academic Publishers, Dordrecht, The Netherlands (1989).

[24] H. Osborn and A.C. Petkou, Implications of conformal invariance in field theories for general dimensions, Annals Phys. 231 (1994) 311 [hep-th/9307010] [INSPIRE].

[25] J.D. Qualls, Lectures on conformal field theory, arXiv:1511.04074 [INSPIRE].

[26] S. Rychkov, EPFL lectures on conformal field theory in $D \geq 3$ dimensions, SpringerBriefs Phys., (2016) [arXiv:1601.05000] [INSPIRE].

[27] A. Chodos and E. Myers, Gravitational contribution to the Casimir energy in Kaluza-Klein theories, Annals Phys. 156 (1984) 412 [INSPIRE].

[28] M.A. Rubin and C.R. Ordonez, Symmetric tensor eigen spectrum of the laplacian on $n$ spheres, J. Math. Phys. 26 (1985) 65 [INSPIRE].

[29] M.A. Rubin and C.R. Ordonez, Eigenvalues and degeneracies for n-dimensional tensor spherical harmonics, UTTG-10-83, (1983) [INSPIRE].

[30] A. Higuchi, Symmetric tensor spherical harmonics on the $N$ sphere and their application to the de Sitter group $\mathrm{SO}(N, 1)$, J. Math. Phys. 28 (1987) 1553 [Erratum ibid. 43 (2002) 6385] [INSPIRE].

[31] A. Ishibashi and R.M. Wald, Dynamics in nonglobally hyperbolic static space-times. 3. Anti-de Sitter space-time, Class. Quant. Grav. 21 (2004) 2981 [hep-th/0402184] [INSPIRE].

[32] J.R. Klauder, Continuous-representation theory. I. Postulates of continuous-representation theory, J. Math. Phys. 4 (1963) 1055.

[33] J.R. Klauder, Continuous-representation theory. II. Generalized relation between quantum and classical dynamics, J. Math. Phys. 4 (1963) 1058.

[34] A.M. Perelomov, Coherent states for arbitrary Lie groups, Commun. Math. Phys. 26 (1972) 222 [INSPIRE].

[35] L.G. Yaffe, Large $N$ limits as classical mechanics, Rev. Mod. Phys. 54 (1982) 407 [InSPIRE]. 
[36] K. Bhaumik and B. Dutta-Roy, The classical nonlinear oscillator and the coherent state, J. Math. Phys. 16 (1975) 1131.

[37] S.M. McRae and E.R. Vrscay, Perturbation theory and the classical limit of quantum mechanics, J. Math. Phys. 38 (1997) 2899.

[38] V. Balasubramanian and P. Kraus, A stress tensor for anti-de Sitter gravity, Commun. Math. Phys. 208 (1999) 413 [hep-th/9902121] [INSPIRE].

[39] S. de Haro, S.N. Solodukhin and K. Skenderis, Holographic reconstruction of space-time and renormalization in the AdS/CFT correspondence, Commun. Math. Phys. 217 (2001) 595 [hep-th/0002230] [INSPIRE].

[40] D. Marolf, W. Kelly and S. Fischetti, Conserved charges in asymptotically (locally) AdS spacetimes, in Springer Handbook of Spacetime, A. Ashtekar and V. Petkov eds., Springer, Berlin, Heidelberg, Germany (2014), pg. 381 [arXiv:1211.6347] [INSPIRE].

[41] J.D. Brown and J.W. York, Jr., Quasilocal energy and conserved charges derived from the gravitational action, Phys. Rev. D 47 (1993) 1407 [gr-qc/9209012] [INSPIRE].

[42] M. Henningson and K. Skenderis, The holographic Weyl anomaly, JHEP 07 (1998) 023 [hep-th/9806087] [INSPIRE].

[43] D. Marolf, Unitarity and holography in gravitational physics, Phys. Rev. D 79 (2009) 044010 [arXiv: 0808.2842] [INSPIRE].

[44] A. Buchel, J. Escobedo, R.C. Myers, M.F. Paulos, A. Sinha and M. Smolkin, Holographic GB gravity in arbitrary dimensions, JHEP 03 (2010) 111 [arXiv: 0911.4257] [INSPIRE].

[45] X.O. Camanho, J.D. Edelstein, J. Maldacena and A. Zhiboedov, Causality constraints on corrections to the graviton three-point coupling, JHEP 02 (2016) 020 [arXiv:1407.5597] [INSPIRE].

[46] N. Afkhami-Jeddi, T. Hartman, S. Kundu and A. Tajdini, Einstein gravity 3-point functions from conformal field theory, JHEP 12 (2017) 049 [arXiv: 1610.09378] [INSPIRE].

[47] M. Botta-Cantcheff, P. Martínez and G.A. Silva, On excited states in real-time AdS/CFT, JHEP 02 (2016) 171 [arXiv: 1512.07850] [INSPIRE].

[48] M. Botta-Cantcheff, P.J. Martínez and G.A. Silva, Interacting fields in real-time AdS/CFT, JHEP 03 (2017) 148 [arXiv:1703.02384] [INSPIRE].

[49] D. Marolf, O. Parrikar, C. Rabideau, A. Izadi Rad and M. Van Raamsdonk, From Euclidean sources to Lorentzian spacetimes in holographic conformal field theories, JHEP 06 (2018) 077 [arXiv:1709.10101] [INSPIRE].

[50] A. Bernamonti, F. Galli, J. Hernandez, R.C. Myers, S.-M. Ruan and J. Simón, First law of holographic complexity, Phys. Rev. Lett. 123 (2019) 081601 [arXiv:1903.04511] [INSPIRE]. 